\title{
Rotating multi-charge spindles and their microstates
}

\author{
Seyed Morteza Hosseini, ${ }^{a}$ Kiril Hristov $^{b, c}$ and Alberto Zaffaroni ${ }^{d, e}$ \\ ${ }^{a}$ Kavli IPMU (WPI), UTIAS, The University of Tokyo, \\ Kashiwa, Chiba 277-8583, Japan \\ ${ }^{b}$ Faculty of Physics, Sofia University, \\ 5 James Bourchier Blvd., Sofia 1164, Bulgaria \\ ${ }^{c}$ INRNE, Bulgarian Academy of Sciences, \\ 72 Tsarigradsko Chaussee, Sofia 1784, Bulgaria \\ ${ }^{d}$ Dipartimento di Fisica, Università di Milano-Bicocca, \\ I-20126 Milano, Italy \\ ${ }^{e}$ INFN, sezione di Milano-Bicocca, \\ I-20126 Milano, Italy \\ E-mail: morteza.hosseini@ipmu.jp, khristov@phys.uni-sofia.bg, \\ alberto.zaffaroni@mib.infn.it
}

ABSTRACT: Some $\mathrm{AdS}_{3} \times M_{7}$ type IIB vacua have been recently proposed to arise from D3branes wrapped on a spindle, a sphere with conical singularities at the poles. We explicitly construct a generalization of these solutions corresponding to a class of electrically charged and rotating supersymmetric black strings in $\operatorname{AdS}_{5} \times S^{5}$ with general magnetic fluxes on the spindle. We then perform a counting of their microstates using the charged Cardy formula. To this purpose, we derive the general form of the anomaly polynomial of the dual $\mathcal{N}=(0,2)$ CFT in two dimensions and we show that it can be obtained via a simple gluing procedure.

Keywords: AdS-CFT Correspondence, Black Holes in String Theory, Conformal Field Theory, Supersymmetric Gauge Theory

ArXiv EPrint: 2104.11249 


\section{Contents}

1 Introduction 1

2 5d spindle horizons from 4d supergravity 3

2.1 The metric on the spindle 4

2.2 The $4 \mathrm{~d} / 5 \mathrm{~d}$ relation 5

2.3 BPS black holes in 4d: the untwisted branch 8

3 Spindle black strings in the stu model $\quad 10$

$\begin{array}{ll}3.1 & \text { Rotating dyonic spindles with equal magnetic charges } \\ \end{array}$

$\begin{array}{lll}3.2 & \text { Static spindles with different magnetic charges } & 14\end{array}$

$\begin{array}{lll}3.3 & \text { Entropy function from gravitational blocks } & 18\end{array}$

4 The charged Cardy formula and the spindle microstates 19

$4.1 \mathcal{A}_{2 \mathrm{~d}}$ for $\mathcal{N}=4$ super Yang-Mills on the spindle 20

4.2 The case with one magnetic charge 23

$\begin{array}{lll}4.3 & \text { The case with arbitrary magnetic charges } & 25\end{array}$

$5 \mathcal{A}_{2 \mathrm{~d}}$ for general $\mathcal{N}=1$ theories on the spindle $\quad 26$

5.1 Integrating the anomaly polynomial 26

5.2 The universal spindles in theories with 8 and 16 supercharges 29

6 Discussion and outlook $\quad 30$

\section{Introduction}

The relation of the Cardy formula [1] to the physics of black holes and black strings is an old subject. In this paper we consider near-horizon solutions of rotating black strings that can be embedded in $\mathrm{AdS}_{5} \times S^{5}$. In five-dimensional language, these are expected to arise as supersymmetric domain walls that interpolate between $\mathrm{AdS}_{5}$ and a near-horizon region consisting of a warped fibration of the Bañados-Teitelboim-Zanelli (BTZ) metric over a two-dimensional compact space. By adding momentum along the string direction and compactifying it on a circle we can obtain a black hole with a smooth near-horizon geometry in four dimensions. We recently constructed various examples of spherical string solutions that can be embedded in $\mathrm{AdS}_{5} \times S^{5}$ (or $\mathrm{AdS}_{7} \times S^{4}$ ) and successfully matched the entropy of the corresponding four-dimensional black holes with a microscopic counting based on the charged Cardy formula [2,3]. We considered rotating and charged generalizations of well-known $\mathrm{AdS}_{3}$ vacua in type IIB and M-theory obtained by compactifying D3- and M5-branes on a sphere with a topological twist [4-7]. 
In this paper we extend our analysis to another class of supersymmetric $\mathrm{AdS}_{3}$ vacua in type IIB string theory which are based on D3-branes wrapped on a spindle, i.e. the one-dimensional complex weighted projective space, $\mathbb{W P}_{\left[n_{1}, n_{2}\right]}^{1}$, which is an orbifold with spherical topology and two conical singularities at the poles [8-12]. An example of this class of solutions was originally found in [8] and later generalized in [9] working in ten dimensions. It can be seen as a ten-dimensional uplift of a $\mathrm{AdS}_{3} \times \mathbb{W P}_{\left[n_{1}, n_{2}\right]}^{1}$ supersymmetric solution of minimal gauged supergravity found in [10] and generalized in [11] to the case of multiple charges. Supersymmetry is realized through a magnetic flux along the spindle but the theory is not topologically twisted [12]. Notably, the full ten-dimensional metric is completely regular, i.e. the apparent conical singularities in five dimensions are smoothed out by the embedding of the spindle inside a seven-dimensional geometry [11, 12]. The authors of $[10,11]$ described these solutions as potential horizons of "unbalanced spherical black rings". More recently the authors of [12] successfully pursued the interpretation in terms of $\mathcal{N}=4$ super Yang-Mills (SYM) theory wrapped on the spindle which gives rise to a two-dimensional $\mathcal{N}=(0,2)$ conformal field theory (CFT). Quite remarkably, despite the presence of singularities, they were able to match the central charge computed holographically with the result obtained from integrating the anomaly polynomial of $\mathcal{N}=4$ SYM on $\mathbb{W P}_{\left[n_{1}, n_{2}\right]}^{1}$.

In the first part of the paper, we generalize these solutions by adding rotation and general electric and magnetic charges. To this purpose, we work in a four-dimensional setting using the recent general construction of supersymmetric dyonic rotating black holes in four-dimensional $\mathcal{N}=2$ gauged supergravity with vector multiplets [13, 14]. Unlike the solutions with a topological twist [4-7] that arise from the twisted branch discussed in [13], the spindle black holes arise from the untwisted branch [14], which also contains the Kerr-Newman black holes in $\mathrm{AdS}_{4}$. These kinds of solutions are usually discarded because of the conical singularities. Once these are allowed, many new solutions naturally appear. In particular, we construct the near-horizon of a family of dyonic rotating black spindles, depending on two independent magnetic fluxes, three electric charges and one angular momentum subject to a constraint. We discuss in detail the conserved electromagnetic charges and angular momentum, as well as the Bekenstein-Hawking entropy of the solutions. Then we uplift them to a five-dimensional gauged supergravity, which is a consistent truncation of type IIB on $\mathrm{AdS}_{5} \times S^{5}$, and, finally, to type IIB.

In the last part of the paper, we successfully match the entropy with a microscopic counting of states using the charged Cardy formula,

$$
\log \rho_{\text {susy }}\left(n_{l}, J_{A}\right) \approx 2 \pi \sqrt{\frac{c_{l}}{6}\left(n_{l}-\frac{c_{l}}{24}-\frac{1}{2}\left(k^{-1}\right)^{A B} J_{A} J_{B}\right)},
$$

for the density of states with energy $n_{l}$ and charges $J_{A}$ of a two-dimensional CFT. To use this formula we need to compute the central charge $c_{l}$ and the levels $k_{A B}$ of the currents in the CFT at large $N$. These can be extracted from the two-dimensional anomaly polynomial, which, in turn, can be obtained by integrating the four-dimensional anomaly polynomial of $\mathcal{N}=4 \mathrm{SYM}$ on the compactification manifold. As for twisted compactifications, we need to include a background for the internal U(1) isometry, which becomes a global symmetry 
in the two-dimensional theory $[3,15,16]$. The two-dimensional anomaly polynomial in the case of the static spindle with a single magnetic flux was derived in [12]. Here, we generalize the derivation to the case of general magnetic charges and of general background fields for the flavor charges. As in [12], the exact two-dimensional $R$-symmetry mixes with the rotational symmetry of the spindle. Interestingly, also in this case, despite the conical singularities, all the anomaly coefficients of the two-dimensional CFT can be extracted from purely four-dimensional physics. For future reference, we also give the expression of the two-dimensional anomaly polynomial for a generic $\mathcal{N}=1 \mathrm{CFT}$.

It is interesting to observe that the two-dimensional anomaly polynomial in the large $N$ limit can be obtained from the gluing formula (5.6). This has a clear counterpart in the proposal for writing entropy functionals for AdS black holes and black strings by gluing gravitational blocks [17] and, given the analogy with holomorphic blocks in quantum field theory (QFT) [18], it could shed further light on the physics of these systems.

The paper is organized as follows. In section 2, we discuss how to obtain supersymmetric spindle solutions from four-dimensional gauged supergravity and set the conventions for the rest of this work. In section 3, we construct the near-horizon of the general family of dyonic rotating black spindles in the stu model. We will focus explicitly on two examples, which will be useful later. The first is a rotating, electrically charged generalization of the static spindle discussed in [12]. The second is a static spindle depending on general magnetic charges under the $\mathrm{U}(1)^{3}$ isometry of $S^{5}$, which was already considered in [12]. In section 4 , we derive the two-dimensional anomaly polynomial of the corresponding $\mathcal{N}=(0,2)$ CFT and match the Bekenstein-Hawking entropy of the solutions previously found with a microscopic counting based on the charged Cardy formula. In section 5, we consider the anomaly polynomial for the compactification of an $\mathcal{N}=1 \mathrm{CFT}$ on the spindle and examine the case of universal spindle solutions of minimal and half-maximal supergravity that can be embedded in most $\mathrm{AdS}_{5}$ compactifications with eight and sixteen supersymmetries, respectively. We conclude with comments and discussion in section 6 .

\section{$2 \quad 5 \mathrm{~d}$ spindle horizons from $4 \mathrm{~d}$ supergravity}

In this section we outline our approach to finding supersymmetric near-horizon geometries in five-dimensional $\mathcal{N}=2$ gauged supergravity that are most generally fibrations of the BTZ metric with the spindle. The solutions that we present explicitly in the next section are generalizations of the direct product solutions of $\mathrm{AdS}_{3}$ with the spindle with purely magnetic charges found in minimal gauged supergravity in [10] and in the gauged stu model in [11]. These solutions were found earlier from a ten-dimensional perspective in $[8,9]$, but our approach leads to a more immediate comparison with [10, 11].

Here, we present a way to generalize the known static near-horizon spindle solutions to include electric charges and rotation. This is done using the connection between the five-dimensional supergravity models we consider and their corresponding four-dimensional reduction. This step requires that we add momentum along the string direction and compactify it to a circle in order to arrive at a smooth near-horizon solution in four dimensions, thus changing the global structure of $\mathrm{AdS}_{3}$ to BTZ. It is precisely this step 
that allows us to add extra electric charges and angular momentum to these solutions, which have eluded the standard classifications of $\mathrm{AdS}_{3}$ solutions in ten dimensions or direct five-dimensional searches.

We first emphasize the new features of the spindle metric that we focus on, before taking a more detailed look at the $4 \mathrm{~d} / 5 \mathrm{~d}$ relation and the explicit $4 \mathrm{~d}$ class of solutions found in [14] that we need here. The explicit results from this preliminary analysis are presented in the next section.

\subsection{The metric on the spindle}

We set our goal to look for the so-called spindle horizons, and therefore need to first describe the characteristics that set apart these horizons when looking for a particular supergravity solution. As described in $[12,19]$, the weighted projective space $\mathbb{W P}_{\left[n_{1}, n_{2}\right]}^{1}$ is topologically a sphere with conical deficit angles $2 \pi\left(1-1 / n_{1,2}\right)$ at the poles with $n_{1} \neq n_{2}$ coprime positive integers. The Euler characteristic of the spindle is then given by

$$
\chi(\Sigma)=\frac{1}{4 \pi} \int_{\Sigma} R_{\Sigma} \mathrm{vol}_{\Sigma}=\frac{1}{n_{1}}+\frac{1}{n_{2}},
$$

where for brevity we introduced the notation $\Sigma=\mathbb{W P}_{\left[n_{1}, n_{2}\right]}^{1}$. We should note that in the math literature this is considered a bad orbifold in the sense that it does not admit a manifold as a covering space. This also means that the spindle can never have a constant curvature metric, making the distinction with other horizon topologies more manifest. ${ }^{1}$

Schematically, up to conformal rescaling and model-dependent constant factors, ${ }^{2}$ the spindle part of the metrics that we are going to consider here has the form

$$
\mathrm{d} s_{\Sigma}^{2} \sim \frac{\mathrm{d} p^{2}}{P(p)}+\frac{P(p)}{p^{2}+P(p)} \mathrm{d} z^{2},
$$

where $z$ is a $\mathrm{U}(1)$ isometry of the full metric (the axis of rotation) and the function $P(p)$ is in the general form

$$
P(p)=s \prod_{a=1}^{n}\left(p-p_{a}\right), \quad p_{a}<p_{a+1} .
$$

Here, $s$ is a choice of overall sign setting the asymptotics, and generically the order of the polynomial is $n=4$ for asymptotically $\mathrm{AdS}_{4}$ solutions ${ }^{3}$ and $n=3$ for asymptotically $\mathrm{AdS}_{5}$ solutions, but various special cases can occur depending on the specific type of solutions, conserved charges, etc. The spindle, like the sphere, is a compact space and therefore the function $P(p)$ must be positive semi-definite and bounded. In practice this leaves very little room for choice: for example in the case $n=2$ the only chance that we find a compact metric is when $s=-1$ and we restrict $p \in\left[p_{1}, p_{2}\right]$. Similarly for $n=4, s=+1$ we must

\footnotetext{
${ }^{1}$ Note however that rotating black holes with spherical horizons in four dimensions also generically do not admit a constant curvature metric, so the deficit angle criterion discussed below remains the ultimate distinction.

${ }^{2}$ See the next section for the explicit solutions and precise constants and details of the metric; they are not important for the general discussion here.

${ }^{3}$ This case is relevant for example for the accelerating and spinning $\mathrm{AdS}_{4}$ black hole spindle in [19].
} 
choose $p \in\left[p_{2}, p_{3}\right]$. Let us now focus on the cubic case that is directly relevant for the spindle strings in five dimensions:

$$
n=3: \quad s=+1, p \in\left[p_{1}, p_{2}\right], \quad \text { or } \quad s=-1, p \in\left[p_{2}, p_{3}\right] .
$$

The two choices are physically equivalent, and with no loss of generality we are going to choose the former case, $s=+1$. Note that all the solutions we present below allow also for a non-compact hyperbolic metric with the choice $n=3, s=+1, p \in\left[p_{3}, \infty\right]$. We leave this potentially interesting parameter space of hyperbolic black strings for a future investigation. ${ }^{4}$

Let us now look at the resulting metric near the poles. Using the coordinate redefinition $R \equiv 2 \sqrt{p-p_{l}}$, we can write the behavior of the metric near the two roots $p=p_{l}, l=1,2$,

$$
\mathrm{d} s_{\Sigma}^{2} \sim \frac{1}{P^{\prime}\left(p_{l}\right)}\left(\mathrm{d} R^{2}+\frac{P^{\prime}\left(p_{l}\right)^{2}}{4 p_{l}^{2}} R^{2} \mathrm{~d} z^{2}\right) .
$$

At each of the poles separately we can always choose the period $\Delta z$ such that we get the metric on the $\mathbb{R}^{2}$ in polar coordinates. If this were possible simultaneously for a single choice of the period $\Delta z$ at the two poles we would find a smooth manifold, i.e. the sphere. In the cubic case however this is only possible if $p_{1}=p_{2}$, which is already inconsistent with the initial requirement that the metric is compact. Therefore, one always finds at least one conical deficit angle, prompting the initial name "unbalanced ring" in $[10,11]$. We can instead obtain the metric on the spindle with two orbifold points of the form $\mathbb{C} / \mathbb{Z}_{n_{1}}$ and $\mathbb{C} / \mathbb{Z}_{n_{2}}$ with two positive coprime integers if we impose that

$$
\frac{P^{\prime}\left(p_{l}\right)^{2}}{4 p_{l}^{2}}=\left(\frac{2 \pi}{n_{l} \Delta z}\right)^{2}
$$

at both poles, thus fixing the period $\Delta z$. This identification is going to be vital for the uplift to ten dimensions where the conical singularities get smoothed out inside the bigger internal space.

Let us finish the present discussion by noting that in a sense the spindle horizons are a generic feature of BPS and thermal black hole/string solutions in AdS. The type of function $P(p)$ and conical deficit angles describing the spindle require no fine tuning of parameters and in this sense are expected to show up in various solutions. It is in fact the smooth spherical horizon that requires an extra condition to be met (namely that $n_{1}=n_{2}$ ), and therefore the spherical case generally requires one additional constraint in the parameter space of solutions in comparison to the spindle.

\subsection{The $4 d / 5 d$ relation}

We are interested in finding solutions to five-dimensional $\mathcal{N}=2$ gauged supergravity coupled to $n_{\mathrm{V}}$ abelian vector multiplets. The Lagrangian is determined from the cubic

\footnotetext{
${ }^{4}$ Above we already imposed that the roots of $P(p)$ are real and non-degenerate since in the cubic case this is the only chance for obtaining a compact metric; in the degenerate case there exist even more exotic possibilities such as non-compact horizons with finite area [20, 21].
} 
prepotential function

$$
\mathcal{F}_{5 \mathrm{~d}}\left(L^{i}\right)=\frac{1}{6} c_{i j k} L^{i} L^{j} L^{k}=1
$$

where the five-dimensional scalars $L^{i}, i=1, \ldots, n_{\mathrm{V}}$, are real and $c_{i j k}$ is a fully symmetric tensor appearing in the Chern-Simons terms, corresponding to the 't Hooft anomaly coefficients of the dual four-dimensional $\mathcal{N}=1 \mathrm{CFT}[22,23]$. The bosonic action is given by

$$
\begin{aligned}
S^{(5)}=\frac{1}{8 \pi G_{\mathrm{N}}^{(5)}} \int_{\mathbb{R}^{4,1}} & {\left[\frac{1}{2} R^{(5)} \star_{5} 1-\frac{1}{2} G_{i j} \mathrm{~d} L^{i} \wedge \star_{5} \mathrm{~d} L^{j}-\frac{1}{2} G_{i j} F^{i} \wedge \star_{5} F^{j}\right.} \\
& \left.-\frac{1}{12} c_{i j k} F^{i} \wedge F^{j} \wedge A^{k}+V \star_{5} 1\right],
\end{aligned}
$$

with $R^{(5)}$ being the Ricci scalar, $F^{i} \equiv \mathrm{d} A^{i}$ is the five-dimensional Maxwell field strength, and $G_{i j}$ can be written in terms of $\mathcal{F}_{5 \mathrm{~d}}$,

$$
G_{i j}=-\left.\frac{1}{2} \partial_{i} \partial_{j} \log \mathcal{F}_{5 \mathrm{~d}}\right|_{\mathcal{F}_{5 \mathrm{~d}}=1} .
$$

The scalar potential can be written in terms of the inverse metric on the scalar manifold as

$$
V(L)=9 V_{i} V_{j}\left(L^{i} L^{j}-\frac{1}{2} G^{i j}\right)
$$

where the constants $V_{i}$ denote the five-dimensional Fayet-Iliopoulos (FI) parameters that specify completely the model together with the tensor $c_{i j k}$. For more details about the five-dimensional conventions see [24].

In the gauged stu model $\left(n_{\mathrm{V}}=3\right)$ that we are going to consider explicitly, the only nonzero intersection numbers are $c_{123}=1$ (and cyclic permutations). The FI parameters are given by

$$
V_{1}=V_{2}=V_{3}=\frac{g_{(5)}}{3},
$$

where $g_{(5)}$ sets the length scale of $\mathrm{AdS}_{5}$. This gauged stu model admits an embedding in $\mathcal{N}=8$ gauged supergravity and an uplift on the $S^{5}$ to ten dimensions [25]. It also admits a truncation to the $s t^{2}$ model $\left(L^{2}=L^{3}\right)$ that can be embedded in minimal $\mathcal{N}=4$ supergravity and thus admits a larger set of string theory embeddings [26-28]. Finally, we could even go to the $t^{3}$ model $\left(L^{1}=L^{2}=L^{3}=1\right)$, which is just minimal $\mathcal{N}=2$ gauged supergravity admitting an uplift on any $\mathrm{SE}_{5}$ and any other $\mathrm{AdS}_{5}$ solution [29].

Reducing the five-dimensional theory along the circle $x^{5}$ one obtains a four-dimensional $\mathcal{N}=2$ gauged supergravity theory based on the prepotential

$$
\mathcal{F}_{4 \mathrm{~d}}\left(X^{\Lambda}\right)=\frac{1}{6} c_{i j k} \frac{X^{1} X^{2} X^{3}}{X^{0}}
$$

where the four-dimensional scalars $X^{\Lambda}=\left(X^{0}, X^{i}\right)$ are complex. The four-dimensional theory contains $n_{\mathrm{V}}$ abelian vector multiplets, parameterizing a special Kähler manifold $\mathcal{M}$ with metric $g_{i \bar{j}}$, besides the gravity multiplet. The following are the rules for reducing the 
bosonic fields $[30-33]:^{5}$

$$
\begin{array}{rlrl}
\mathrm{d} s_{(5)}^{2} & =\mathrm{e}^{2 \varphi} \mathrm{d} s_{(4)}^{2}+\mathrm{e}^{-4 \varphi}\left(\mathrm{d} x^{5}-\sqrt{2} A_{(4)}^{0}\right)^{2}, & & \mathrm{~d} x^{5}=\mathrm{d} w, \\
A_{(5)}^{i} & =\sqrt{2} A_{(4)}^{i}+\operatorname{Re} z^{i}\left(\mathrm{~d} x^{5}-\sqrt{2} A_{(4)}^{0}\right), & & \\
L^{i} & =\mathrm{e}^{2 \varphi} \llbracket \mathrm{m} z^{i}, & \mathrm{e}^{-6 \varphi}=\frac{1}{6} c_{i j k}
\end{array}
$$

Here, $\varphi$ is the Kaluza-Klein (KK) scalar (called dilaton), $\mathrm{d} s_{(4)}^{2}$ is the four-dimensional line element, $A_{(4)}^{\Lambda}, \Lambda=0, \ldots, n_{\mathrm{V}}$, are the four-dimensional abelian gauge fields and $z^{i}=X^{i} / X^{0}$. The resulting four-dimensional bosonic action is then given by

$$
\begin{aligned}
S^{(4)}=\frac{1}{8 \pi G_{\mathrm{N}}^{(4)}} \int_{\mathbb{R}^{3,1}} & {\left[\frac{1}{2} R^{(4)} \star_{4} 1+\frac{1}{2} \llbracket \mathrm{m} \mathcal{N}_{\Lambda \Sigma} F^{\Lambda} \wedge \star_{4} F^{\Sigma}+\frac{1}{2} \operatorname{Re} \mathcal{N}_{\Lambda \Sigma} F^{\Lambda} \wedge F^{\Sigma}\right.} \\
& \left.-g_{i \bar{j}} D z^{i} \wedge \star_{4} D \bar{z}^{\bar{j}}-V(z, \bar{z}) \star_{4} 1\right],
\end{aligned}
$$

where $V(z, \bar{z})$ is the scalar potential of the theory,

$$
V(z, \bar{z})=g_{\Lambda} g_{\Sigma}\left(U^{\Lambda \Sigma}-3 e^{\mathcal{K}} \bar{X}^{\Lambda} X^{\Sigma}\right)
$$

with $g_{\Lambda}$ being the four-dimensional constant FI parameters. See more details about the other four-dimensional supergravity quantities appearing in the action in e.g. [35]. Just like in five dimensions, the four-dimensional action is completely specified by the choice of prepotential and FI parameters. The five-dimensional gauged stu model therefore gives rise to the cubic prepotential (2.12) with $c_{123}=1$ (and cyclic permutations). In the fermionic sector we do a direct Kaluza-Klein reduction (as opposed to Scherk-Schwarz, see [36, 37]) and therefore find the following four-dimensional FI parameters

$$
g_{0}=0, \quad g_{1}=g_{2}=g_{3}=g_{(4)},
$$

where, in our conventions,

$$
g_{(4)}=\sqrt{2} g_{(5)}
$$

We can therefore look for a spindle near-horizon geometry of the type $\mathrm{AdS}_{2}$ fibered over $\Sigma$ satisfying the criteria from section 2.1, which upon uplift becomes a fibration of BTZ over $\Sigma$. This would constitute a near-horizon geometry of a spindle black string in the original five-dimensional $\mathcal{N}=2$ gauged $s t u$ model specified above. Notice, that we need the extra circle $w$ not to be fibered over the spindle, such that we keep the same spindle geometry in five dimensions. We therefore impose

$$
\int_{\Sigma} \mathrm{d} A_{(4)}^{0}=0
$$

meaning that we need a vanishing magnetic charge $m^{0}=0$. This is an additional requirement that we impose on the four-dimensional solutions we consider next. Note that $m^{0} \neq 0$ instead leads in general to a Lens space horizon in five dimensions, which will be explored elsewhere.

\footnotetext{
${ }^{5}$ In our conventions the four-dimensional and five-dimensional gauge fields are related by $A_{(5)}^{i}=\sqrt{2} A_{(4)}^{i}$. We make this choice for convenience to land directly at the conventions used in [14] based on the fourdimensional BPS equations in [34].
} 


\subsection{BPS black holes in 4d: the untwisted branch}

We have now effectively reduced our initial problem from five to four dimensions, the upshot being that the general supersymmetric near-horizon solutions in four-dimensional $\mathcal{N}=2$ gauged supergravity with abelian vector multiplets have already been written down exhaustively in the presence of the most general set of electromagnetic charges and rotation. The BPS solutions we discuss here are derived by solving the first-order integrability conditions following from a timelike isometry [34, 38], which were further rewritten in the formalism and conventions we follow here in $[13,14]$. In particular, one finds two disjoint branches of solutions - the twisted ${ }^{6}$ and the untwisted ${ }^{7}$ solutions. The twisted branch in the model described above was used in [2] to generalize the static black strings of [6] to the case with electric charges and rotation. It is straightforward to see that the requirement $m^{0}=0$ for the twisted branch leads to a quadratic form of the function $P(p)$ in $(2.2)$ and eventually one finds that the twisted branch can only lead to spherical and not to spindle horizons for five-dimensional black strings. Therefore, we turn our attention completely to the untwisted, or Kerr-Newman-like branch of solutions that lead to a cubic polynomial for $P(p)$. The situation here is in fact reversed and we only find spindle (as opposed to spherical) horizons with non-vanishing area.

Referring the reader for all technical details to the original reference, [14], here we summarize the main features of the untwisted near-horizons needed for the presentation of the solutions. We take the following ansatz for the four-dimensional spacetime,

$$
\mathrm{d} s_{4}^{2}=-e^{2 U}(\mathrm{~d} t+\omega)^{2}+e^{-2 U} \mathrm{~d} s_{3}^{2},
$$

with a base metric

$$
\mathrm{d} s_{3}^{2}=e^{2 \sigma}\left(\frac{\mathrm{d} p^{2}}{P(p)}+\frac{\mathrm{d} r^{2}}{Q(r)}\right)+P(p) Q(r) \mathrm{d} z^{2},
$$

where

$$
e^{2 \sigma}=r^{2} P(p)+p^{2} Q(r),
$$

and $\omega$ is the rotation one-form living on the base space. The physical scalars $z^{i}$ together with the scalar factor $e^{U}$ are more conveniently packaged in the symplectic notation

$$
\left\{X^{\Lambda} ; F_{\Lambda}\right\}=e^{-2 U} \mathcal{R}+\mathrm{i} \mathcal{I}, \quad \mathcal{R}=-\frac{1}{2 I_{4}(\mathcal{I})} I_{4}^{\prime}(\mathcal{I})=-\frac{1}{2} e^{4 U} I_{4}^{\prime}(\mathcal{I})
$$

where $F_{\Lambda} \equiv \frac{\partial \mathcal{F}_{4 \mathrm{~d}}\left(X^{\Lambda}\right)}{\partial X^{\Lambda}}$ and in this parametrization the symplectic vector $\mathcal{I}$ determines both the real and the imaginary part of the physical scalars. In the second equality we also used the so-called quartic invariant, $I_{4}$, which is indispensable for solving the BPS equations. The equations to be solved are given in [14, (12)-(14)]. More technical aspects of the formalism are summarized in [13] and the appendices of $[17,40]$. In the stu model with

\footnotetext{
${ }^{6}$ The most general twisted near-horizon geometry was written down in [13] generalizing [39, 40].

${ }^{7}$ The most general untwisted near-horizon geometry was written down in [14] generalizing the supersymmetric limit of the Kerr-Newman-AdS 4 black hole.
} 
cubic prepotential coming from the 5d reduction, (2.12), the quartic invariant and all its derivatives are uniquely defined by the equality

$$
I_{4}\left(\left\{p^{\Lambda} ; q_{\Lambda}\right\}\right)=4 q_{0} p^{1} p^{2} p^{3}-\left(p^{i} q_{i}\right)^{2}+2 \sum_{i<j}^{3} q_{i} p^{i} q_{j} p^{j}-p^{0}\left(4 q_{1} q_{2} q_{3}+p^{0}\left(q_{0}\right)^{2}+2 q_{0} p^{i} q_{i}\right) .
$$

Finally, the four-dimensional gauge fields $A$ (or $\left\{A^{\Lambda} ; A_{\Lambda}\right\}$, including both electric and magnetic gauge fields) can also be expressed in terms of the three-dimensional gauge fields $\mathcal{A}=\left\{\mathcal{A}^{\Lambda} ; \mathcal{A}_{\Lambda}\right\}$ on the base space,

$$
\left\{F^{\Lambda} ; G_{\Lambda}\right\} \equiv \mathrm{d} A=\mathrm{d}(\zeta(\mathrm{d} t+\omega))+\mathcal{F}=\mathrm{d}(\zeta(\mathrm{d} t+\omega))+\mathrm{d} \mathcal{A}, \quad \zeta=\mathcal{R}-G p r
$$

Here, $G$ denotes the symplectic vector of gauging parameters defining the Lagrangian and obtained from the $5 \mathrm{~d}$ reduction

$$
G=\{0 ; 0, g, g, g\}
$$

Note that here we omitted the subscript (4) in comparison with (2.16) for simplicity and will consistently do so below as long as we stay in four dimensions. It is important here to note that the specific cubic prepotential and gauging parameters lead to the identity

$$
I_{4}(G)=0
$$

which is always satisfied for models coming from a $5 \mathrm{~d}$ reduction as it signifies that there

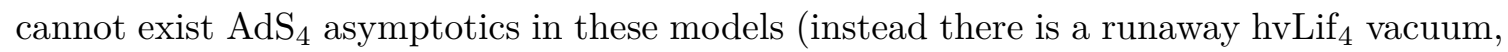
see more in [13]).

In the $\mathrm{AdS}_{2} \times \Sigma$ near-horizon geometry conformal invariance requires $Q(r)=R_{0}^{2} r^{2}$, where $R_{0}$ is a free constant, and

$$
e^{2 \sigma}=r^{2} e^{2 \sigma_{0}}, \quad e^{2 U}=r^{2} e^{2 U_{0}}, \quad \omega=\frac{1}{r} \omega_{0},
$$

where $\sigma_{0}, U_{0}$ and $\omega_{0}$ are functions of $p$.

Taking an ansatz for $\mathcal{I}$ in terms of a symplectic vector $\mathcal{H}$ polynomial in $p$,

$$
\mathcal{I}=e^{-2 \sigma} \mathcal{H}, \quad \mathcal{H}=r\left(\frac{\mathcal{C}}{\Xi}+p \mathcal{C}_{1}+p^{2} \mathcal{C}_{2}+p^{3} \mathcal{C}_{3}\right)
$$

with a free constant $\Xi$, the full set of BPS equations are solved in terms of the free constant symplectic vector $\mathcal{C}$. The symplectic vectors $\mathcal{C}_{1,2,3}$ determining the scalars, metric functions and the three-dimensional gauge fields $\mathcal{A}$ are then explicitly fixed by $\mathcal{C}$, the gauging vector $G$ and the quartic invariant $I_{4}$ using $[14,(22)]$. The rotation one-form reads

$$
\omega_{0}=-\frac{P(p) e^{-2 \sigma_{0}}}{2 \Xi}\left\langle G, I_{4}^{\prime}(\mathcal{C})\right\rangle
$$

and thus vanishes in the special case that the symplectic product between the symplectic vectors $G$ and $I_{4}^{\prime}(\mathcal{C})$ vanishes. In the explicit examples below this indeed corresponds to the static spindle string solutions in [10-12]. 
Finally, let us again focus on the form of the function $P(p)$, given by

$$
P(p)=e^{-2 \sigma_{0}}-R_{0}^{2} p^{2}=\Xi^{-1}\left(1+2\langle G, \mathcal{C}\rangle p+k_{2} p^{2}+\frac{1}{2}\left\langle I_{4}^{\prime}(G), I_{4}^{\prime}(\mathcal{C})\right\rangle p^{3}\right),
$$

where we defined $k_{2} \equiv\langle G, \mathcal{C}\rangle^{2}-\frac{1}{4} I_{4}(\mathcal{C}, \mathcal{C}, G, G)-\Xi R_{0}^{2}$. Note that in general $P(p)$ also has a quartic power proportional to $I_{4}(G)$ that vanishes identically in the model of interest. Lead by an interest in smooth spherical horizons, [14] imposed in addition that $\langle G, \mathcal{C}\rangle=$ $\left\langle I_{4}^{\prime}(G), I_{4}^{\prime}(\mathcal{C})\right\rangle=0$, which is where our present analysis diverges. The only requirement that we impose on the a priori arbitrary constant vector $\mathcal{C}$ is dictated by the condition of vanishing fibration of the additional circle with the spindle, (2.18). We therefore arrive at the most general solution in the gauged stu model corresponding to spindle black strings,

$$
\mathcal{C}=\left\{0, \frac{1}{2 g a_{1}}, \frac{1}{2 g a_{2}}, \frac{1}{2 g a_{3}} ; b_{0}, b_{1}, b_{2}, b_{3}\right\},
$$

where $a_{1,2,3}, b_{0,1,2,3}$ are arbitrary constants and we inserted the factors of $g$ for later convenience. The choice of $\mathcal{C}$ in turn fixes all the conserved electromagnetic charges, rotation and entropy of the spindle horizons, which we evaluate explicitly in a couple of special examples. Note also that we can rescale some of the parameters by a change of coordinates. In the following examples, we use this freedom to set $R_{0}$ and $\Xi$ to a convenient value without loss of generality on the physical observables and then analyze the metric on the spindle according to the considerations in section 2.1.

\section{Spindle black strings in the stu model}

So far we outlined a general procedure for finding spindle horizons via a passage through four-dimensional horizons. We formally wrote down the most general solution in the gauged stu model corresponding to a spindle black string horizon in (2.31), parametrized by a number of free constants. We now turn to discuss the physics behind the solution in a couple of different limiting cases.

First, we look at the equal magnetic charges case (setting $a_{1}=a_{2}=a_{3}$ in (2.31)), allowing for arbitrary electric charges and rotation. Then, we consider carefully the static case with different magnetic charges but vanishing electric charges (setting $b_{1}=b_{2}=b_{3}=0$ in (2.31)). This solution was already discussed in [11]. In both cases we elaborate on the spindle metric and evaluate the conserved electromagnetic charges and angular momentum, as well as the Bekenstein-Hawking entropy. In the first case, the full ten-dimensional regularity was already shown in $[10,12]$ for a general $\mathrm{SE}_{5}$ uplift in agreement with [8]. We discuss the ten-dimensional solution in the second case that only allows an $S^{5}$ uplift, where we again see that the conical singularities are smoothed out [11], as was seen also directly in ten dimensions [9].

At the end of this section we also discuss how to obtain the resulting entropy from the procedure of gluing gravitational blocks generalizing [17] to spindle horizons, which will turn out to mimic the way that the anomaly polynomial of the holographic dual CFT factorizes at the poles of the spindle. 


\subsection{Rotating dyonic spindles with equal magnetic charges}

In this section we present a rotating and electrically charged generalization of the solution in $[10,12]$. As described in the previous section, we first present the solution in fourdimensional language where it corresponds to the near-horizon of a rotating black hole with electric and magnetic charges. To have a real and positive entropy, we introduce a momentum along the compactifying circle which shows up in four dimensions as an electric charge $q_{0}$. Similarly to $[2,3]$, upon uplifting to five dimensions, the solution becomes a fibration of BTZ and the spindle. We allow for arbitrary electric charges $q_{i}, i=1,2,3$, but we restrict to equal magnetic charges, for simplicity.

Solving the BPS equations $[14,(22)]$ with the following choice of the symplectic vector $\mathcal{C}$,

$$
\mathcal{C}=\left\{0, \frac{1}{2 a g}, \frac{1}{2 a g}, \frac{1}{2 a g} ; b_{0}, b_{1}, b_{2}, b_{3}\right\},
$$

and a convenient choice for the parameters $R_{0}$ and $\Xi,^{8}$ leads to a metric specified by the following functions

$$
\begin{array}{rlrl}
Q(r) & =r^{2}, & P(p) & =\frac{4}{27}(p+a)^{3}-p^{2}, \\
e^{2 \sigma_{0}} & =\frac{4}{27}(p+a)^{3}, & \omega_{0}=-\frac{a}{27 g}\left(1-\frac{27}{4} \frac{p^{2}}{(p+a)^{3}}\right) \sum_{i=1}^{3} b_{i}, \\
e^{2 U_{0}}=\frac{4 g^{\frac{3}{2}}(p+a)^{3}}{\sqrt{8 b_{0}(p+a)^{3}-a g \Pi\left(4(p+a)^{3}-a p^{2}\right)-2 g(a p)^{2} \sum_{i=1}^{3} b_{i}^{2}}}
\end{array}
$$

where we defined

$$
\Pi=\sum_{i=1}^{3} b_{i}^{2}-2\left(b_{1} b_{2}+b_{2} b_{3}+b_{1} b_{3}\right) .
$$

The regularity of the metric can be directly checked following the procedure in section 2.1. We however decide to first change to the coordinates of [12] for a simpler comparison. Using the variable

$$
p=3 y-a,
$$

we can write the polynomial $P$ as

$$
P(y)=4 y^{3}-(3 y-a)^{2}=-\left(3 y+2 y^{3 / 2}-a\right)\left(3 y-2 y^{3 / 2}-a\right) .
$$

Near the zeros of $P(y)$, we have conical singularities and it is impossible to obtain a smooth metric. We can instead obtain a metric with two orbifold singular points of the form $\mathbb{R}^{1,1} \times \mathbb{C} / \mathbb{Z}_{n_{1}}$ and $\mathbb{R}^{1,1} \times \mathbb{C} / \mathbb{Z}_{n_{2}}$, where $n_{1}$ and $n_{2}$ are two positive coprime integers. Consider the part of metric described by the coordinates $(y, z)$. Near a zero $y_{l}$ of $P(y)$, using $R=2 \sqrt{y-y_{l}}$, we can write

$$
\mathrm{d} s_{\Sigma}^{2} \sim \frac{1}{P^{\prime}\left(y_{l}\right)}\left(\mathrm{d} R^{2}+\frac{P^{\prime}\left(y_{l}\right)^{2}}{36 e^{2 \sigma_{0}\left(y_{l}\right)}} R^{2} \mathrm{~d} z^{2}\right) .
$$

\footnotetext{
${ }^{8}$ Here, we set $R_{0}=1$ and $\Xi=\frac{27}{4 a^{3}}$.
} 
Choosing the period $\Delta z$ for $z$ such that

$$
\frac{P^{\prime}\left(y_{l}\right)^{2}}{36 e^{2 \sigma_{0}\left(y_{l}\right)}} \equiv\left(\frac{2 \pi}{\Delta z n_{l}}\right)^{2}, \quad \text { for } \quad l=1,2,
$$

we obtain a metric with deficit angles $2 \pi / n_{1}$ and $2 \pi / n_{2}$ at $y_{1}$ and $y_{2}$, respectively. Taking the zeros such that $3 y_{l}-2 y_{l}^{3 / 2}-a=0$ for $l=1,2$, we obtain, by consistency,

$$
a=\frac{\left(n_{1}-n_{2}\right)^{2}\left(2 n_{1}+n_{2}\right)^{2}\left(n_{1}+2 n_{2}\right)^{2}}{4\left(n_{1}^{2}+n_{2} n_{1}+n_{2}^{2}\right)^{3}}, \quad \Delta z=4 \pi \frac{n_{1}^{2}+n_{2} n_{1}+n_{2}^{2}}{3 n_{1} n_{2}\left(n_{1}+n_{2}\right)},
$$

and

$$
y_{1}=\frac{\left(n_{1}-n_{2}\right)^{2}\left(2 n_{1}+n_{2}\right)^{2}}{4\left(n_{1}^{2}+n_{2} n_{1}+n_{2}^{2}\right)^{2}}, \quad y_{2}=\frac{\left(n_{1}-n_{2}\right)^{2}\left(n_{1}+2 n_{2}\right)^{2}}{4\left(n_{1}^{2}+n_{2} n_{1}+n_{2}^{2}\right)^{2}} .
$$

With $y \in\left[y_{1}, y_{2}\right]$ we obtain a positive definite metric on the orbifold $\Sigma=\mathbb{W P}_{\left[n_{1}, n_{2}\right]}^{1}$. Observe that $y_{1}<y_{2}$ for $n_{1}<n_{2}$.

The symplectic vector of gauge fields $\mathcal{A}=\left\{0, \mathcal{A}^{i} ; \mathcal{A}_{\Lambda}\right\}$ is given by

$$
\begin{aligned}
& \mathcal{A}^{i}=\frac{1}{6 g} \frac{3 y-a}{y} \mathrm{~d} z, \quad \text { for } \quad i=1,2,3, \\
& \mathcal{A}_{0}=(3 y-a)\left[b_{0}+\frac{g a(3 y-a)}{4(3 y)^{3}}\left(a^{2}\left(b_{1}+b_{2}+b_{3}\right)^{2}-9 \Pi y^{2}\right)\right] \mathrm{d} z, \\
& \mathcal{A}_{i}=\frac{a(3 y-a)}{4(3 y)^{3}}\left(36 y^{2} b_{i}-\left(9 y^{2}-a^{2}\right)\left(b_{1}+b_{2}+b_{3}\right)\right) \mathrm{d} z, \quad \text { for } \quad i=1,2,3 .
\end{aligned}
$$

Notice that $\mathcal{A}$ are just the components of the gauge fields on the base. The full expression for the gauge fields is given in (2.24) and contains components along $\mathrm{d} t$ that are too long to be reported.

We can now evaluate the conserved charges. The electromagnetic charges read

$$
\Gamma=\frac{1}{4 \pi} \int \mathcal{F}=\left\{m^{\Lambda} ; q_{\Lambda}\right\}
$$

with

$$
\begin{aligned}
m^{0} & =0, \quad m^{i}=\frac{1}{6 g}\left(\frac{1}{n_{1}}-\frac{1}{n_{2}}\right), \quad \text { for } \quad i=1,2,3 \\
q_{i} & =\frac{\left(n_{1}-n_{2}\right)^{3}\left(2 n_{1}+n_{2}\right)^{2}\left(n_{1}+2 n_{2}\right)^{2}}{12^{2} n_{1} n_{2}\left(n_{1}^{2}+n_{2} n_{1}+n_{2}^{2}\right)^{4}}\left(\left(n_{1}-n_{2}\right)^{2} \sum_{j=1}^{3} b_{j}-12\left(n_{1}^{2}+n_{1} n_{2}+n_{2}^{2}\right) b_{i}\right) .
\end{aligned}
$$

There is also a non-zero $q_{0}$ but its expression is not illustrative and we shall not present its explicit form here. Notice that the $R$-symmetry gauge field

$$
A^{R}=\frac{g}{2} \sum_{i=1}^{3} A^{i}
$$

has a flux

$$
\frac{1}{2 \pi} \int_{\Sigma} F^{R}=\frac{1}{2}\left(\frac{1}{n_{1}}-\frac{1}{n_{2}}\right),
$$


along the spindle. Since the gauge field $A^{R}$ is the one appearing in the covariant derivative of the gravitino, we see that supersymmetry is realized with a mechanism analogous to the one discussed in [12]. In particular, the components of $A^{R}$ along the spindle in the gauge (3.17) precisely coincide with those in [12].

The angular momentum can be computed via the Komar integral associated to the spacelike Killing vector $\xi=\partial_{\phi}$,

$$
\mathcal{J}=-\frac{1}{8 \pi} \int_{\Sigma}\left(\star_{4} \mathrm{~d} \xi+2\left(\xi \cdot A^{\Lambda}\right) \mathrm{d} A_{\Lambda}\right)
$$

where $\phi=2 \pi z / \Delta z$ is the angular coordinate on the spindle with period $2 \pi$. The above equation is the symplectically covariant generalization of the formula presented recently in [19, appendix E]. As already noticed there, the angular momentum evaluated at the horizon is not gauge invariant and depends crucially on the choice of pure gauge that one can add to the electric gauge fields. We employ the gauge transformation $A^{\Lambda} \rightarrow A^{\Lambda}+A_{G}^{\Lambda}$,

$$
A_{G}^{0}=0, \quad A_{G}^{i}=-\frac{1}{3 g} \mathrm{~d} z, \quad \text { for } \quad i=1,2,3,
$$

so that at the poles we find

$$
A^{i}\left(y_{l}\right)=-\frac{1}{3 g n_{l}} \mathrm{~d} \phi, \quad \text { for } \quad i=1,2,3, \quad \text { and } \quad l=1,2,
$$

as will be justified later. The angular momentum $\mathcal{J}$ is then given by

$$
\mathcal{J}=\frac{1}{72 g} \frac{\left(n_{1}-n_{2}\right)^{3}\left(n_{1}+n_{2}\right)\left(2 n_{1}+n_{2}\right)^{2}\left(n_{1}+2 n_{2}\right)^{2}}{n_{1}^{2} n_{2}^{2}\left(n_{1}^{2}+n_{2} n_{1}+n_{2}^{2}\right)^{3}} \sum_{i=1}^{3} b_{i} .
$$

It is also interesting to observe that the conserved charges obey the following constraint

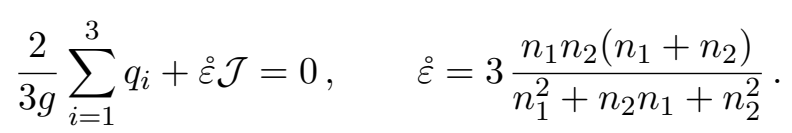

Constraints among charges are common for supersymmetric AdS black holes and occur both for twisted and Kerr-Newman ones.

Finally, the Bekenstein-Hawking entropy reads

$$
\begin{aligned}
S_{\mathrm{BH}} & =\frac{\text { Area }}{4 G_{\mathrm{N}}^{(4)}} \\
& =\frac{3}{8 G_{\mathrm{N}}^{(4)}}\left(\frac{3 g}{2}\right)^{-\frac{3}{2}} \sqrt{b_{0}+a g\left(2\left(b_{1} b_{2}+b_{2} b_{3}+b_{3} b_{1}\right)-\frac{1}{2}\left(1+\frac{a}{27}\right)\left(b_{1}+b_{2}+b_{3}\right)^{2}\right)}\left(y_{2}-y_{1}\right) \Delta z \\
& =\frac{\pi}{g G_{\mathrm{N}}^{(4)}} \sqrt{\frac{\left(n_{2}-n_{1}\right)^{3}}{18 g n_{1} n_{2}\left(n_{1}^{2}+n_{1} n_{2}+n_{2}^{2}\right)}\left(q_{0}-\frac{1}{2} \sum_{A, B=1}^{2}\left(q_{A}-q_{3}\right)\left(\mathrm{k}^{-1}\right)_{A B}\left(q_{B}-q_{3}\right)-\frac{\mathcal{J}^{2}}{2 \mathrm{k}}\right)},
\end{aligned}
$$

where

$$
\mathrm{k}_{A B}=\frac{1}{6 g}\left(\frac{1}{n_{1}}-\frac{1}{n_{2}}\right)\left(\begin{array}{ll}
2 & 1 \\
1 & 2
\end{array}\right), \quad \mathrm{k}=-\frac{1}{(3 g)^{3}}\left(\frac{1}{n_{1}^{3}}-\frac{1}{n_{2}^{3}}\right)
$$


The solution can be uplifted to five dimensions using the formula (2.13). When $\mathcal{J}=0$ and $q_{i}=0, i=1,2,3$, by rescaling the time coordinate, we obtain ${ }^{9}$

$$
\mathrm{d} s_{5}^{2}=\frac{1}{g_{(5)}^{2}}\left(\frac{4 y}{9} \mathrm{~d} s_{\mathrm{BTZ}}^{2}+\frac{y}{P(y)} \mathrm{d} y^{2}+\frac{P(y)}{(6 y)^{2}} \mathrm{~d} z^{2}\right),
$$

with the extremal BTZ metric

$$
\mathrm{d} s_{\text {BTZ }}^{2}=\frac{1}{4}\left(\frac{\mathrm{d} r^{2}}{r^{2}}-r^{2} \mathrm{~d} t^{2}\right)+r_{+}^{2}\left(\mathrm{~d} w+\frac{r}{2 r_{+}} \mathrm{d} t\right)^{2},
$$

where $r_{+}=\frac{1}{2}(3 g)^{3 / 2} \sqrt{b_{0}}$ is related to the four-dimensional electric charge $q_{0}$. Since BTZ is metrically equivalent to $\mathrm{AdS}_{3}$, we can replace $\mathrm{d} s_{\mathrm{BTZ}}^{2}$ with $\mathrm{d} s_{\mathrm{AdS}_{3}}^{2}$ in (3.22) and recover the solution found in [12], corresponding to a two-dimensional CFT with central charge

$$
c^{\mathrm{CFT}}=\frac{N^{2}}{3} \frac{\left(n_{2}-n_{1}\right)^{3}}{n_{1} n_{2}\left(n_{1}^{2}+n_{1} n_{2}+n_{2}^{2}\right)} .
$$

In the solution (3.22) there is a momentum along the BTZ circle, which is necessary to have a black hole without naked singularities in four dimensions. From the field theory point of view, we are considering states in the $\mathrm{CFT}_{2}$ with energy proportional to $q_{0}$.

For non-vanishing angular momentum and electric charges, the five-dimensional metric is more complicated and it is a non-trivial fibration of the spindle and the BTZ metric. The physical interpretation is however simple. The solution is dual to an ensemble of states of the $\mathrm{CFT}_{2}$ with energy proportional to $q_{0}$ and conserved abelian charges $q_{i}$ and $\mathcal{J}$. And, indeed, the entropy (3.20) is strongly reminiscent of the charged Cardy formula (1.1) that captures the density of states of a $\mathrm{CFT}_{2}$. We confirm this interpretation in section 4 .

\subsection{Static spindles with different magnetic charges}

We now look more closely at the case of three different magnetic charges and vanishing electric charges $q_{i}=0\left(b_{1}=b_{2}=b_{3}=0\right.$ in $\left.(2.31)\right)$. This is the four-dimensional version of the solution presented in [11] with extra momentum along the string direction. We first write the solution in four dimensions and then uplift it to five dimensions, where it becomes a warped product of BTZ and the spindle, and, later on, to a smooth solution in ten dimensions.

We parametrize the symplectic vector $\mathcal{C}$ in the following way,

$$
\mathcal{C}=\left\{0, \frac{1}{2 g a_{1}}, \frac{1}{2 g a_{2}}, \frac{1}{2 g a_{3}} ; \frac{b_{0}}{(2 g)^{3} a_{1} a_{2} a_{3}}, 0,0,0\right\}
$$

which leads to a static solution with ${ }^{10}$

$$
\begin{aligned}
Q(r) & =a_{1} a_{2} a_{3} r^{2}, & P(p) & =\prod_{i=1}^{3}\left(p+a_{i}\right)-p^{2} a_{1} a_{2} a_{3}, \\
e^{2 \sigma_{0}} & =\prod_{i=1}^{3}\left(p+a_{i}\right), & e^{2 U_{0}} & =\frac{1}{2}(2 g)^{3} e^{\sigma_{0}} \sqrt{\frac{a_{1} a_{2} a_{3}}{b_{0}}} .
\end{aligned}
$$

\footnotetext{
${ }^{9}$ Recall that in our conventions $g_{(5)}=g_{(4)} / \sqrt{2}$ and we denoted $g_{(4)} \equiv g$. We also used the expression $\zeta=r\left\{-\frac{1}{2 b_{0}}, 0,0,0 ; 0, g a, g a, g a\right\}$, valid for zero angular momentum and zero electric charges, to evaluate the time component of the gauge field $A^{0}$.

${ }^{10}$ Here, we choose for convenience $R_{0}=\frac{1}{\sqrt{\Xi}}$ and $\Xi=\frac{1}{a_{1} a_{2} a_{3}}$.
} 
To find a metric on the spindle, we follow the procedure in section 2.1. The cubic polynomial $P(p)$ has three real positive roots which we denote by $p_{l}, l=1,2,3$. Near $p_{l}$, the $(p, z)$ part of the metric behaves as

$$
\mathrm{d} s_{\Sigma}^{2} \sim \frac{1}{P^{\prime}\left(p_{l}\right)}\left(\frac{\mathrm{d} p^{2}}{p-p_{l}}+\frac{a_{1} a_{2} a_{3}}{e^{2 \sigma_{0}\left(p_{l}\right)}}\left(p-p_{l}\right) P^{\prime}\left(p_{l}\right)^{2} \mathrm{~d} z^{2}\right)
$$

Using $R=2 \sqrt{p-p_{l}}$, we obtain

$$
\mathrm{d} s_{\Sigma}^{2} \sim \frac{1}{P^{\prime}\left(p_{l}\right)}\left(\mathrm{d} R^{2}+\frac{a_{1} a_{2} a_{3} P^{\prime}\left(p_{l}\right)^{2}}{4 e^{2 \sigma_{0}\left(p_{l}\right)}} R^{2} \mathrm{~d} z^{2}\right) .
$$

Choosing the period $\Delta z$ for $z$ such that

$$
\frac{a_{1} a_{2} a_{3} P^{\prime}\left(p_{l}\right)^{2}}{4 e^{2 \sigma_{0}\left(p_{l}\right)}} \equiv\left(\frac{2 \pi}{\Delta z n_{l}}\right)^{2}, \quad \text { for } \quad l=1,2,
$$

where $\left(n_{1}, n_{2}\right)$ are arbitrary coprime positive integers with $n_{1}<n_{2}$, we obtain conical singularities with deficit angles $2 \pi / n_{1}$ and $2 \pi / n_{2}$ at $p_{1}$ and $p_{2}$, respectively. The roots of the cubic polynomial $P(p)$ can then be written as

$$
\begin{aligned}
& p_{1}=-\frac{1}{18 n_{1} n_{2}\left(n_{1}+n_{2}\right)}\left(\mathcal{L}+2\left(n_{1}-n_{2}\right) \sqrt{\frac{2 \pi}{\Delta z}}\right)\left(\mathcal{L}-2\left(2 n_{1}+n_{2}\right) \sqrt{\frac{2 \pi}{\Delta z}}\right), \\
& p_{2}=-\frac{1}{18 n_{1} n_{2}\left(n_{1}+n_{2}\right)}\left(\mathcal{L}+2\left(n_{1}-n_{2}\right) \sqrt{\frac{2 \pi}{\Delta z}}\right)\left(\mathcal{L}+2\left(n_{1}+2 n_{2}\right) \sqrt{\frac{2 \pi}{\Delta z}}\right), \\
& p_{3}=-\frac{1}{18 n_{1} n_{2}\left(n_{1}+n_{2}\right)}\left(\mathcal{L}-2\left(2 n_{1}+n_{2}\right) \sqrt{\frac{2 \pi}{\Delta z}}\right)\left(\mathcal{L}+2\left(n_{1}+2 n_{2}\right) \sqrt{\frac{2 \pi}{\Delta z}}\right) .
\end{aligned}
$$

Here, we also included the third root of $P(p)$ for completeness and, for the ease of notation, we defined

$$
\mathcal{L}=\sqrt{4\left(n_{1}^{2}+n_{1} n_{2}+n_{2}^{2}\right) \frac{2 \pi}{\Delta z}+6\left(a_{1}+a_{2}+a_{3}-a_{1} a_{2} a_{3}\right) n_{1} n_{2}\left(n_{1}+n_{2}\right)} .
$$

We choose $p \in\left[p_{1}, p_{2}\right]$ to obtain a positive definite metric on the orbifold $\Sigma=\mathbb{W P}_{\left[n_{1}, n_{2}\right]}^{1}$. Observe that $p_{1}<p_{2}$ for $n_{1}<n_{2}$.

The symplectic vector of gauge fields on the base is given by

$$
\mathcal{A}=\left\{0, \frac{a_{1} a_{2} a_{3}}{2 g} \frac{p}{p+a_{1}} \mathrm{~d} z, \frac{a_{1} a_{2} a_{3}}{2 g} \frac{p}{p+a_{2}} \mathrm{~d} z, \frac{a_{1} a_{2} a_{3}}{2 g} \frac{p}{p+a_{3}} \mathrm{~d} z ; \frac{b_{0}}{(2 g)^{3}} p \mathrm{~d} z, 0,0,0\right\},
$$

and the complete expression for the gauge fields is given by (2.24) with

$$
\zeta=r\left\{-\frac{4 g^{3} a_{1} a_{2} a_{3}}{b_{0}}, 0,0,0 ; 0, g a_{1}, g a_{2}, g a_{3}\right\} .
$$

The corresponding electromagnetic charges

$$
\Gamma=\frac{1}{4 \pi} \int_{\Sigma} \mathcal{F}=\left\{m^{\Lambda} ; q_{\Lambda}\right\},
$$


are given by

$$
\begin{array}{ll}
m^{0}=0, & q_{0}=\frac{b_{0}}{32 \pi g^{3}}\left(p_{2}-p_{1}\right) \Delta z, \\
m^{i}=\frac{a_{1} a_{2} a_{3}\left(p_{2}-p_{1}\right)}{8 \pi g} \frac{a_{i}}{\left(p_{1}+a_{i}\right)\left(p_{2}+a_{i}\right)} \Delta z, & q_{i}=0, \quad \text { for } \quad i=1,2,3 .
\end{array}
$$

Note the following useful relations among the magnetic charges

$$
\begin{aligned}
\sum_{i=1}^{3} m^{i} & =\frac{1}{2 g}\left(\frac{1}{n_{1}}-\frac{1}{n_{2}}\right) \\
\prod_{i=1}^{3} m^{i} & =p_{3}^{2}\left(\frac{1}{8 \pi g}\left(p_{2}-p_{1}\right) \Delta z\right)^{3}, \\
m^{1} m^{2}+m^{2} m^{3}+m^{1} m^{3} & =\frac{\left(p_{1}-p_{3}\right)\left(p_{2}-p_{3}\right)+p_{1} p_{2} p_{3}^{2}}{p_{1} p_{2}}\left(\frac{1}{8 \pi g}\left(p_{2}-p_{1}\right) \Delta z\right)^{2} \\
& =-\frac{1}{(2 g)^{2}}\left(\frac{1}{n_{1} n_{2}}-\frac{a_{1} a_{2} a_{3}}{4 \pi}\left(\frac{1}{n_{1}}+\frac{1}{n_{2}}\right) \Delta z\right) .
\end{aligned}
$$

The first equation in (3.36) ensures again that the $R$-symmetry field $A^{R}=g \sum_{i=1}^{3} A^{i} / 2$ has a flux

$$
\frac{1}{2 \pi} \int_{\Sigma} F^{R}=\frac{1}{2}\left(\frac{1}{n_{1}}-\frac{1}{n_{2}}\right)
$$

along the spindle, which is necessary to enforce supersymmetry [12]. The electric charge can be also rewritten as

$$
q_{0}=\frac{b_{0}}{(2 g)^{2}} \frac{m^{1} m^{2} m^{3}}{\frac{1}{(2 g)^{2} n_{1} n_{2}}+m^{1} m^{2}+m^{2} m^{3}+m^{1} m^{3}} .
$$

With this information we can evaluate the Bekenstein-Hawking entropy

$$
\begin{aligned}
S_{\mathrm{BH}} & =\frac{\text { Area }}{4 G_{\mathrm{N}}^{(4)}}=\frac{\sqrt{b_{0}}}{2(2 g)^{3} G_{\mathrm{N}}^{(4)}}\left(p_{2}-p_{1}\right) \Delta z \\
& =\frac{\pi}{g G_{\mathrm{N}}^{(4)}} \sqrt{\frac{m^{1} m^{2} m^{3}}{\frac{1}{(2 g)^{2} n_{1} n_{2}}+m^{1} m^{2}+m^{2} m^{3}+m^{1} m^{3}} q_{0}} .
\end{aligned}
$$

The spindle black string, using the uplift formula (2.13), can be recast as

$$
\begin{aligned}
& \mathrm{d} s_{5}^{2}=\frac{1}{4 g_{(5)}^{2}}\left(\frac{4 e^{\frac{2}{3} \sigma_{0}}}{a_{1} a_{2} a_{3}} \mathrm{~d} s_{\mathrm{BTZ}}^{2}+\frac{e^{\frac{2}{3} \sigma_{0}}}{P(p)} \mathrm{d} p^{2}+\frac{a_{1} a_{2} a_{3} P(p)}{e^{\frac{4}{3} \sigma_{0}}} \mathrm{~d} z^{2}\right), \\
& A_{(5)}^{i}=\rho^{i}(p) \mathrm{d} z \equiv \frac{a_{1} a_{2} a_{3}}{2 g_{(5)}} \frac{p}{p+a_{i}} \mathrm{~d} z, \quad L^{i}=\frac{e^{\frac{2}{3} \sigma_{0}}}{p+a_{i}}, \quad \text { for } \quad i=1,2,3,
\end{aligned}
$$

with the extremal BTZ metric given in (3.23) and $r_{+}=\frac{\sqrt{b_{0}}}{2 \sqrt{2}}$. Note that the dilaton is given by

$$
e^{2 \varphi}=2 g_{(5)} \sqrt{\frac{2 a_{1} a_{2} a_{3}}{b_{0}}} e^{-\frac{1}{3} \sigma_{0}} .
$$


Since the BTZ is locally equivalent to $\mathrm{AdS}_{3}$, we can replace $\mathrm{d} s_{\mathrm{BTZ}}^{2}$ with $\mathrm{d} s_{\mathrm{AdS}_{3}}^{2}$ in (3.40) and find a generalization of the $\mathrm{AdS}_{3}$ solution found in [12] depending on general magnetic charges and discussed in $[9,11]$. We compute the central charge of the corresponding $\mathrm{CFT}_{2}$ and explicitly match the entropy (3.39) with the charged Cardy formula in section 4. Our solution reduces to the one in [12] for $a_{1}=a_{2}=a_{3} \cdot{ }^{11}$

We can uplift the solution to ten dimensions using [25, (2.1)]

$$
\mathrm{d} s_{10}^{2}=\sqrt{\Delta} \mathrm{d} s_{5}^{2}+\frac{1}{g_{(5)}^{2} \sqrt{\Delta}} \sum_{i=1}^{3} \frac{1}{L^{i}}\left(\mathrm{~d} \mu_{i}^{2}+\mu_{i}^{2}\left(\mathrm{~d} \varphi_{i}+g_{(5)} A_{(5)}^{i}\right)^{2}\right),
$$

where $\Delta=\sum_{i=1}^{3} L^{i} \mu_{i}^{2}$, and

$$
\mu_{1}=\sin \theta, \quad \mu_{2}=\cos \theta \sin \psi, \quad \mu_{3}=\cos \theta \cos \psi,
$$

so that

$$
\sum_{i=1}^{3} \mu_{i}^{2}=1, \quad \mathrm{~d} s_{S^{5}}^{2}=\sum_{i=1}^{3}\left(\mathrm{~d} \mu_{i}^{2}+\mu_{i}^{2} \mathrm{~d} \varphi_{i}^{2}\right) .
$$

The metric (3.42) can then also be rewritten as

$$
\begin{aligned}
\mathrm{d} s_{10}^{2}= & \frac{\sqrt{\Delta} e^{\frac{2}{3} \sigma_{0}}}{4 g_{(5)}^{2}}\left[\frac{4}{a_{1} a_{2} a_{3}} \mathrm{~d} s_{\mathrm{BTZ}}^{2}+\frac{\mathrm{d} p^{2}}{P}+\frac{a_{1} a_{2} a_{3} P}{e^{2 \sigma_{0}}}\left(1+\frac{\sqrt{2} g_{(5)} p e^{\frac{2}{3} \sigma_{0}}}{P \Delta} \sum_{a=1}^{3} \rho^{a} \mu_{a}^{2}\right) \mathrm{D} z^{2}\right] \\
& +\frac{1}{g_{(5)}^{2} \sqrt{\Delta}} \mathrm{d} s_{B_{5}}^{2},
\end{aligned}
$$

where

$$
\begin{aligned}
\mathrm{D} z & \equiv \mathrm{d} z+\frac{2 \sqrt{2} p e^{\frac{2}{3} \sigma_{0}}}{\Delta\left(P+e^{2 \sigma_{0}}\right)} \sum_{a=1}^{3} \mu_{a}^{2} \mathrm{~d} \varphi_{a}, \\
\mathrm{~d} s_{B_{5}}^{2} & =\sum_{a=1}^{3} \frac{1}{L^{a}}\left(\mathrm{~d} \mu_{a}^{2}+\mu_{a}^{2} \mathrm{~d} \varphi_{a}^{2}\right)+\frac{P-e^{2 \sigma_{0}}}{\Delta\left(P+e^{2 \sigma_{0}}\right)}\left(\sum_{a=1}^{3} \mu_{a}^{2} \mathrm{~d} \varphi_{a}\right)^{2},
\end{aligned}
$$

which allows to see the transverse seven-dimensional metric as a fibration of the angular variable $z$ over a six-dimensional base.

In the ten-dimensional metric the $\mathrm{U}(1)^{3}$ torus in $S^{5}$ is non-trivially fibered over the spindle with Chern numbers determined by $m^{i}$. The regularity of the ten-dimensional solution has been explicitly checked in $[8,12]$ for $a_{1}=a_{2}=a_{3}$ and in $[9,11]$ for the general case. In the case of equal magnetic charges, the Reeb direction $\psi=\phi_{1}+\phi_{2}+\phi_{3}$ is fibered over the spindle parameterized by $(p, z)$ with connection $2 A$, where (3.14) holds. This gives a Lens space $S^{3} / \mathbb{Z}_{\left(n_{2}-n_{1}\right) / 3}$ fibration over $\mathbb{P}^{2} .{ }^{12}$ When $n_{1}$ and $n_{2}$ are relatively prime and

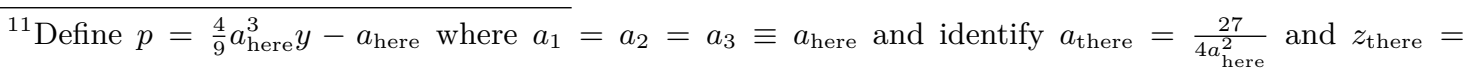
$a_{\text {here }}^{3} z_{\text {here. }}$

${ }^{12}$ The total space of the fibration over the weighted projective space $\mathbb{W P}_{\left[n_{1}, n_{2}\right]}^{1}$ with Chern number $\frac{1}{2 \pi} \int F=r /\left(n_{1} n_{2}\right)$ is the Lens space $S^{3} / \mathbb{Z}_{r}$ where $\mathbb{Z}_{r}$ acts as $\left(z_{1}, z_{2}\right) \rightarrow\left(e^{2 \pi i n_{1} / r} z_{1}, e^{2 \pi i n_{2} / r} z_{2}\right)$ on $S^{3}=\left\{\left(z_{1}, z_{2}\right) \in \mathbb{C}_{2}:\left|z_{1}\right|^{2}+\left|z_{2}\right|^{2}=1\right\}$ - see for example appendix A in [19]. The extra factor of 3 comes from the $6 \pi$ periodicity of $\psi$.
} 
$n_{1}-n_{2}$ is a multiple of 3 , the fibration is regular $[8,12]$. In the general case, the Reeb direction is still fibered on the spindle with the same Chern number, which is fixed by supersymmmetry. In addition, the flavor symmetry directions $\phi_{1}-\phi_{3}$ and $\phi_{2}-\phi_{3}$ are further fibered over the base. It follows from the analysis done in $[9,11]$ that the metric is still regular, for example, if $2 g n_{1} n_{2} m^{i} \in \mathbb{Z}$ [11], which just imposes further quantization conditions on the flavor magnetic charges $m_{1}-m_{3}$ and $m_{2}-m_{3}$. We leave the full analysis of the quantization conditions for the future.

\subsection{Entropy function from gravitational blocks}

In this section we show that can write an entropy functional for the spindle solution by gluing gravitational blocks, thus confirming the general prescription introduced in [17].

It has been shown in [17] that all known entropy functionals for $\mathrm{AdS}_{4}$ black holes and dimensional reduction of $\mathrm{AdS}_{5}$ black strings can be written as

$$
\mathcal{I}\left(p^{\Lambda}, \lambda^{\Lambda}, \varepsilon\right) \equiv \frac{\pi}{4 G_{\mathrm{N}}^{(4)}}\left(\sum_{\sigma=1}^{2} \mathcal{B}\left(X_{(\sigma)}^{\Lambda}, \varepsilon_{(\sigma)}\right)-2 \mathrm{i} \lambda^{\Lambda} q_{\Lambda}-2 \varepsilon \mathcal{J}\right)
$$

where $\lambda^{\Lambda}$ and $\varepsilon$ are the chemical potentials conjugated to the electric charges $q_{\Lambda}$ and the angular momentum $\mathcal{J}$, respectively, and the gravitational block

$$
\mathcal{B}\left(X^{\Lambda}, \varepsilon\right) \equiv-\frac{\mathcal{F}_{4 \mathrm{~d}}\left(X^{\Lambda}\right)}{\varepsilon},
$$

is constructed in terms of the prepotential $\mathcal{F}_{4 \mathrm{~d}}$ of the corresponding gauged supergravity. The functional $\mathcal{I}$ must be extremized with respect to the chemical potentials $\lambda^{\Lambda}$ and $\varepsilon$ subject to a constraint and the extremum value is the entropy of the black hole. The details of the gluing functions $\left(X_{(\sigma)}^{\Lambda}, \varepsilon_{(\sigma)}\right)$ and of the constraint depend on the type of black hole, either twisted or of Kerr-Newman type, and are discussed in [17]. The construction is the gravitational dual of the realization of three-dimensional supersymmetric partition functions obtained by gluing holomorphic blocks [18], and fusing partition functions on hemispheres. The two factors $\sigma=1$ and $\sigma=2$ correspond, in this language, to the North and South hemisphere of the horizon geometry $S^{2}$.

In the case at hand, the prepotential is given by

$$
\mathcal{F}_{4 \mathrm{~d}}=\frac{X^{1} X^{2} X^{3}}{X^{0}}
$$

and the sphere is replaced by the orbifold $\Sigma=\mathbb{W P}_{\left[n_{1}, n_{2}\right]}^{1}$. The gluing that works for the spindle is

$$
\begin{array}{rlrl}
X^{0} & =\lambda^{0}, \\
X_{(1)}^{i} & =\lambda^{i}-\mathrm{i} \varepsilon\left(m^{\Lambda}-\frac{1}{6 g} \chi\right), & \varepsilon_{(1)} & =\varepsilon, \\
X_{(2)}^{i} & =\lambda^{i}+\mathrm{i} \varepsilon\left(m^{\Lambda}+\frac{1}{6 g} \chi\right), & \varepsilon_{(2)} & =-\varepsilon,
\end{array}
$$


where $\chi$ is the Euler number of spindle, given in (2.1), and the chemical potentials are constrained to satisfy

$$
g_{\Lambda} \lambda^{\Lambda}=g \sum_{i=1}^{3} \lambda^{i}=2 .
$$

One can explicitly check that the entropy of both the rotating and magnetically charged spindle black holes, (3.20) and (3.39), can be obtained by extremizing the entropy functional (3.47).

We can shed some light on the form of the gluing (3.50) by considering first the case of equal magnetic charges $m^{i}=\frac{1}{6 g}\left(\frac{1}{n_{1}}-\frac{1}{n_{2}}\right)$ with $i=1,2,3$. The gluing of the components $i=1,2,3$ simplifies to

$$
\begin{array}{ll}
X_{(1)}^{i}=\lambda^{i}+\mathrm{i} \varepsilon \frac{1}{3 g n_{2}}, & \varepsilon_{(1)}=\varepsilon, \\
X_{(2)}^{i}=\lambda^{i}+\mathrm{i} \varepsilon \frac{1}{3 g n_{1}}, & \varepsilon_{(2)}=-\varepsilon .
\end{array}
$$

We see that supersymmetry requires shifting $\lambda^{i}$ by a quantity proportional to the value of $R$-symmetry gauge field at the two hemispheres, see (3.17). This is a natural generalization of what happens for twisted black holes [17], which motivated our ansatz. In the general case, we can write

$$
\begin{array}{ll}
X_{(1)}^{i}=\lambda^{i}-\mathrm{i} \varepsilon\left(s^{i}-\frac{1}{3 g n_{2}}\right), & \varepsilon_{(1)}=\varepsilon, \\
X_{(2)}^{i}=\lambda^{i}+\mathrm{i} \varepsilon\left(s^{i}+\frac{1}{3 g n_{1}}\right), & \varepsilon_{(2)}=-\varepsilon,
\end{array}
$$

where, using (3.36), we parameterized

$$
m^{i}=s^{i}+\frac{1}{6 g}\left(\frac{1}{n_{1}}-\frac{1}{n_{2}}\right), \quad \text { for } \quad i=1,2,3,
$$

with $\sum_{i=1}^{3} s^{i}=0$. The $s^{i}$ can be seen as magnetic fluxes for the flavor symmetries of $\mathcal{N}=4$ SYM. They enter in the gluing formula in analogy with the examples discussed in [17].

The form of the gluing (3.50) should follow from the gluing of holomorphic blocks in a field theory computation. The identification and evaluation of the supersymmetric index relevant for the spindle is left for future work. It is not completely obvious what partition function we should consider in the presence of singularities. We can however observe that the form of the gluing (3.50) suggests that the relevant index is obtained by considering two hemisphere partition functions with the insertion of background magnetic fluxes $\frac{1}{3 g n_{1}}$ and $\frac{1}{3 g n_{2}}$, respectively, and gluing them together in the spirit of [18].

We will see the interpretation of the gluing in terms of the field theory anomaly polynomial in the next section.

\section{The charged Cardy formula and the spindle microstates}

In this section we provide a microscopic counting of the states of the black spindle using the charged Cardy formula. 
The black spindle horizon solutions discussed in this paper are dual to an ensemble of states of a $(0,2)$ supersymmetric CFT (SCFT) with energy $L_{0}=n_{l}$ and charges $J_{A}$ under a set of abelian symmetries. The density of supersymmetric states can be derived from the modular transformation of the elliptic genus and it is given by the charged Cardy formula [3]

$$
\log \rho_{\text {susy }}\left(n_{l}, J_{A}\right) \approx 2 \pi \sqrt{\frac{c_{l}}{6}\left(n_{l}-\frac{c_{l}}{24}-\frac{1}{2}\left(k^{-1}\right)^{A B} J_{A} J_{B}\right)},
$$

where $c_{l}$ is the left-moving central charge and $k_{A B}$ is the matrix of levels of the abelian currents

$$
k_{A B}=-\operatorname{Tr} \gamma_{3} J_{A} J_{B} .
$$

All information needed to evaluated the Cardy formula can be extracted from the twodimensional anomaly polynomial $\mathcal{A}_{2 \mathrm{~d}}$. In particular, the levels coincide (up to a sign) with the 't Hooft anomaly coefficients, $k_{A B}=-\mathcal{A}_{A B}$, defined as ${ }^{13}$

$$
\mathcal{A}_{2 \mathrm{~d}}=\frac{1}{2} \mathcal{A}_{A B} c_{1}\left(F^{A}\right) c_{1}\left(F^{B}\right)+\ldots
$$

The exact $R$-symmetry of the two-dimensional CFT is a linear combination of the abelian symmetries, $R=\sum \stackrel{\circ}{\delta}_{A} J_{A}$ that can be found by extremizing the trial central charge

$$
c_{r}\left(\delta_{A}\right)=3 \operatorname{Tr} \gamma_{3} R(\delta)^{2}=3 \mathcal{A}_{A B} \delta_{A} \delta_{B},
$$

where $R(\delta)=\sum \delta_{A} J_{A}$, with respect to the mixing parameters $\delta_{A}$. The restriction of the anomaly polynomial to the exact $R$-symmetry thus reads

$$
\mathcal{A}_{2 \mathrm{~d}}=\frac{c_{r}}{6} c_{1}(R)^{2},
$$

where $c_{r}=c_{r}(\stackrel{\circ}{\delta}) \equiv c^{\mathrm{CFT}}$ is the exact central charge. Since we work in the holographic regime, $c=c_{l}=c_{r}$.

We now compute the anomaly polynomial for the CFTs dual to the spindle solution and match the charged Cardy formula with the gravitational entropy.

\section{1 $\mathcal{A}_{2 \mathrm{~d}}$ for $\mathcal{N}=4$ super Yang-Mills on the spindle}

The $\mathcal{N}=(0,2)$ SCFT is obtained by compactifying a set of $N$ D3-branes on the spindle, and we expect to read off the $2 \mathrm{~d}$ anomaly polynomial from the integration of the $4 \mathrm{~d}$ anomaly polynomial of $\mathcal{N}=4 \mathrm{SYM}$ on $\Sigma=\mathbb{W P}_{\left[n_{1}, n_{2}\right]}^{1}$ in the presence of magnetic charges for the $\mathrm{U}(1)^{3} \subset \mathrm{SO}(6)$ global symmetries of the four-dimensional theory. The two-dimensional theory has an extra abelian symmetry, in addition to the $\mathrm{U}(1)^{3}$ inherited from the four-dimensional parent theory, that arises from the $\mathrm{U}(1)$ isometry of the spindle. The corresponding conserved charge is what we would call angular momentum from the higher-dimensional perspective. As noticed in [12], the isometry along the spindle mixes in

\footnotetext{
${ }^{13} \mathrm{As}$ in [3], we use notations where supersymmetry is realized in the anti-holomorphic sector and the $2 \mathrm{~d}$ chirality matrix $\gamma_{3}$ is taken to be positive on anti-holomorphic fermionic movers. We also choose the signs in such a way that the level matrix $k_{A B}$ in a unitary theory is positive definite for holomorphic currents.
} 
a non-trivial way with the $R$-symmetry of the two-dimensional theory and we need to take it properly into account. The inclusion of the symmetries coming from the internal geometry in the anomaly polynomial of the lower-dimensionsional CFT has been extensively discussed in $[3,15,16]$ in the case of $S^{2}$. The generalization to the spindle was discussed in [12]. Here we need to further generalize it to the case of arbitrary charges.

Consider $\mathcal{N}=4 \mathrm{SYM}$ on $\Sigma$. We use a basis of the $\mathrm{U}(1)^{3} \subset \mathrm{SO}(6)_{R}$ symmetry assigning charge +1 to chiral superfields $\Phi_{1,2,3}$, respectively; we call their generators and field strengths as $Q_{1,2,3}$ and $F_{1,2,3}$, respectively. The $4 \mathrm{~d}$ anomaly polynomial in the large $N$ limit, for the gauge group $\mathrm{SU}(N)$, is

$$
\mathcal{A}_{4 \mathrm{~d}}=\frac{N^{2}}{2} c_{1}\left(F_{1}\right) c_{1}\left(F_{2}\right) c_{1}\left(F_{3}\right) .
$$

The gravity solution corresponds to the situation where we turn on background gauge fields $A_{i}=\rho_{i}(p) \mathrm{d} \phi$ on the spindle with magnetic fluxes

$$
\frac{1}{2 \pi} \int \rho_{i}^{\prime}(p) \mathrm{d} p \mathrm{~d} \phi=\rho_{i}\left(p_{2}\right)-\rho_{i}\left(p_{1}\right)=\mathfrak{p}^{i}, \quad \text { for } \quad i=1,2,3,
$$

where, for convenience, we normalize the angle $\phi=2 \pi z / \Delta z$ to have period $2 \pi$. As in [12], the supersymmetry constraint (3.37) requires a flux $\frac{1}{2}\left(\frac{1}{n_{1}}-\frac{1}{n_{2}}\right)$ for the $R$-symmetry which in our notations translate to ${ }^{14}$

$$
\sum_{i=1}^{3} \mathfrak{p}^{i}=\frac{1}{n_{1}}-\frac{1}{n_{2}}
$$

We need to pay attention to the choice of gauge. By a gauge transformation, we can always add additive constants to the functions $\rho_{i}(p)$. We work in the gauge where

$$
\rho_{i}\left(p_{2}\right)=\frac{1}{2}\left(\mathfrak{p}^{i}-\frac{1}{3} \chi\right), \quad \rho_{i}\left(p_{1}\right)=\frac{1}{2}\left(-\mathfrak{p}^{i}-\frac{1}{3} \chi\right) .
$$

In particular, the $R$-symmetry background field satisfies

$$
A_{\phi}^{R}\left(p_{2}\right)=-\frac{1}{2 n_{2}}, \quad A_{\phi}^{R}\left(p_{1}\right)=-\frac{1}{2 n_{1}} .
$$

As argued in [12], this choice of gauge ensures that the Killing spinors are independent of $\phi{ }^{15}$ Notice that this is the same gauge used to compute $\mathcal{J}$ in section 3.1 (see (3.17)). The functions $\rho_{i}(p)$ can be read off from section 3.2, after normalization and a gauge transformation, but their explicit forms will not be important in the following.

In order to compute the two-dimensional anomaly polynomial, we also turn on background fields $A_{R}$ and $A_{J}$ probing the $R$-symmetry and the internal U(1) isometry, respectively,

$$
A_{i}=\Delta_{i} A_{R}+\rho_{i}(p)\left(\mathrm{d} \phi+A_{J}\right), \quad \text { for } \quad i=1,2,3,
$$

\footnotetext{
${ }^{14}$ The field theory background fields $A_{i}$ are identified with $g_{(4)} A_{(4)}^{i}=g_{(5)} A_{(5)}^{i}$ on the gravity side.

${ }^{15}$ This requirement only fixes the gauge for the $R$-symmetry. We could use a different gauge for the flavor symmetries, under which the Killing spinors are neutral. This ambiguity can be reabsorbed in a shift of the chemical potentials $\Delta_{i}$ in the trial function $c_{r}\left(\epsilon, \Delta_{i}\right)$ and leads to the same physical prediction for the central charge. Notice however that a change of gauge also leads to a redefinition of the charge associated with the internal isometry. The same is true in gravity, see (3.15).
} 
with curvature

$$
F_{i}=\Delta_{i} F_{R}+\rho_{i}^{\prime}(p) \mathrm{d} p\left(\mathrm{~d} \phi+A_{J}\right)+\rho_{i}(p) F_{J}, \quad \text { for } \quad i=1,2,3,
$$

where now $A_{R}$ and $A_{J}$ are fields in the two-dimensional theory and we have embedded the $2 \mathrm{~d} \mathrm{U}(1)_{R}$ symmetry in the direction $\Delta_{i} Q_{i}$ with $\sum_{i=1}^{3} \Delta_{i}=2$. We can now substitute this expression into (4.6) and integrate it over the spindle to obtain the two-dimensional anomaly polynomial

$$
\mathcal{A}_{2 \mathrm{~d}}=\int_{\Sigma} \mathcal{A}_{4 \mathrm{~d}}
$$

as a function of the background fields $A_{R}$ and $A_{J}$. All integrals in $p$ can be explicitly done and we obtain

$$
\begin{aligned}
\mathcal{A}_{2 \mathrm{~d}}=\frac{N^{2}}{2} & {\left[\frac{1}{2} c_{1}\left(F_{R}\right)^{2} \sum_{i \neq j \neq k} \Delta_{i} \Delta_{j}\left[\rho_{k}\left(p_{2}\right)-\rho_{k}\left(p_{1}\right)\right]\right.} \\
& \left.+\frac{1}{2} c_{1}\left(F_{R}\right) c_{1}(J) \sum_{i \neq j \neq k} \Delta_{i}\left[\rho_{j}\left(p_{2}\right) \rho_{k}\left(p_{2}\right)-\rho_{j}\left(p_{1}\right) \rho_{k}\left(p_{1}\right)\right]\right] \\
& +c_{1}(J)^{2}\left[\rho_{1}\left(p_{2}\right) \rho_{2}\left(p_{2}\right) \rho_{3}\left(p_{2}\right)-\rho_{1}\left(p_{1}\right) \rho_{2}\left(p_{1}\right) \rho_{3}\left(p_{1}\right)\right] .
\end{aligned}
$$

It is interesting to observe that the previous expression can be recast as a sum over fixed points

$$
\mathcal{A}_{2 \mathrm{~d}}=\frac{N^{2}}{2}\left[\frac{1}{c_{1}(J)} \prod_{i=1}^{3}\left(\Delta_{i} c_{1}\left(F_{R}\right)+\rho_{i}\left(p_{2}\right) c_{1}(J)\right)+\frac{1}{\left(-c_{1}(J)\right)} \prod_{i=1}^{3}\left(\Delta_{i} c_{1}\left(F_{R}\right)+\rho_{i}\left(p_{1}\right) c_{1}(J)\right)\right],
$$

of the internal $U(1)$ isometry.

Using (4.9), the $2 \mathrm{~d}$ anomaly polynomial can be compactly written as a gluing formula

$$
\mathcal{A}_{2 \mathrm{~d}}=\frac{16}{27 c_{1}(J)}\left(a_{4 \mathrm{~d}}\left(\Delta_{i}^{(1)}\right)-a_{4 \mathrm{~d}}\left(\Delta_{i}^{(2)}\right)\right)
$$

with

$$
\Delta_{i}^{(1)}=\Delta_{i} c_{1}\left(F_{R}\right)+\frac{c_{1}(J)}{2}\left(\mathfrak{p}^{i}-\frac{1}{3} \chi\right), \quad \Delta_{i}^{(2)}=\Delta_{i} c_{1}\left(F_{R}\right)-\frac{c_{1}(J)}{2}\left(\mathfrak{p}^{i}+\frac{1}{3} \chi\right) .
$$

Note that (4.16) becomes a quadratic polynomial in $c_{1}\left(F_{R}\right)$ and $c_{1}(J)$, after taking the sum over fixed points. Here, $a_{4 \mathrm{~d}}\left(\Delta_{i}\right)$ is the $4 \mathrm{~d}$ trial central charge in the large $N$ limit, which for $\mathcal{N}=4 \mathrm{SYM}$ reads

$$
a_{4 \mathrm{~d}}\left(\Delta_{i}\right)=\frac{27}{32} N^{2} \Delta_{1} \Delta_{2} \Delta_{3} .
$$

The equations (4.17) are the field theory counterparts of the gravitational gluing (3.50) for the components $i=1,2,3$. 


\subsection{The case with one magnetic charge}

The anomaly polynomial of the two-dimensional theory obtained by compactifying $\mathcal{N}=4$ SYM on the spindle with equal magnetic fluxes

$$
\mathfrak{p}^{1}=\mathfrak{p}^{2}=\mathfrak{p}^{3}=\frac{1}{3}\left(\frac{1}{n_{1}}-\frac{1}{n_{2}}\right),
$$

is then given by

$$
\begin{aligned}
\mathcal{A}_{2 \mathrm{~d}}=\frac{2 a_{4 \mathrm{~d}}}{27} & \left(9\left(\Delta_{1} \Delta_{2}+\Delta_{1} \Delta_{3}+\Delta_{2} \Delta_{3}\right)\left(\frac{1}{n_{1}}-\frac{1}{n_{2}}\right) c_{1}\left(F_{R}\right)^{2}\right. \\
& \left.+\left(\frac{1}{n_{1}^{3}}-\frac{1}{n_{2}^{3}}\right) c_{1}(J)^{2}-6\left(\frac{1}{n_{1}^{2}}-\frac{1}{n_{2}^{2}}\right) c_{1}\left(F_{R}\right) c_{1}(J)\right) .
\end{aligned}
$$

Here, $a_{4 \mathrm{~d}}=\frac{N^{2}}{4}$ is the exact central charge of $\mathcal{N}=4 \mathrm{SYM}$ in the large $N$ limit and the chemical potentials $\Delta_{i}, i=1,2,3$, for the $\mathrm{U}(1)^{3} \subset \mathrm{SO}(6)_{R}$ are constrained by

$$
\sum_{i=1}^{3} \Delta_{i}=2
$$

This expression coincides with $[12,(30)]$ after setting $\Delta_{1}=\Delta_{2}=\Delta_{3}=\frac{2}{3}$.

We see that $J$ mixes non-trivially with the $\mathrm{U}(1)^{3}$ symmetries of $\mathcal{N}=4 \mathrm{SYM}$. A convenient to way extract the trial central charge is to allow a mixing $c_{1}(J)=\epsilon c_{1}\left(F_{R}\right)$ and then compute $c_{r}\left(\epsilon, \Delta_{i}\right)=6 \mathcal{A}_{2 \mathrm{~d}} / c_{1}\left(F_{R}\right)^{2}$ :

$$
c_{r}\left(\Delta_{i}, \epsilon\right)=\frac{4 a_{4 \mathrm{~d}}}{9}\left[9\left(\Delta_{1} \Delta_{2}+\Delta_{1} \Delta_{3}+\Delta_{2} \Delta_{3}\right)\left(\frac{1}{n_{1}}-\frac{1}{n_{2}}\right)+\left(\frac{1}{n_{1}^{3}}-\frac{1}{n_{2}^{3}}\right) \epsilon^{2}-6\left(\frac{1}{n_{1}^{2}}-\frac{1}{n_{2}^{2}}\right) \epsilon\right] \text {. }
$$

This has to be extremized over the set of chemical potentials $\left(\epsilon, \Delta_{i}\right)$, under the constraint (4.21). We obtain the critical points

$$
\stackrel{\circ}{\epsilon}=\frac{3 n_{1} n_{2}\left(n_{1}+n_{2}\right)}{n_{1}^{2}+n_{1} n_{2}+n_{2}^{2}}, \quad \stackrel{\circ}{\Delta}_{i}=\frac{2}{3}, \quad \text { for } \quad i=1,2,3 .
$$

We can then read off the exact central charge of the two-dimensional CFT [12]

$$
c^{\mathrm{CFT}}=\frac{4 a_{4 \mathrm{~d}}}{3} \frac{\left(n_{2}-n_{1}\right)^{3}}{n_{1} n_{2}\left(n_{1}^{2}+n_{1} n_{2}+n_{2}^{2}\right)} .
$$

We can define two independent flavor charges, $K_{1}=Q_{1}-Q_{3}$ and $K_{2}=Q_{2}-Q_{3}$, and we can extract their matrix level $k_{A B}, A, B=1,2$, as well as the level $k$ of the $\mathrm{U}(1)$ rotational symmetry using (4.3)

$$
k_{A B}=\frac{2 a_{4 \mathrm{~d}}}{3}\left(\frac{1}{n_{1}}-\frac{1}{n_{2}}\right)\left(\begin{array}{ll}
2 & 1 \\
1 & 2
\end{array}\right), \quad k=-\frac{4 a_{4 \mathrm{~d}}}{27}\left(\frac{1}{n_{1}^{3}}-\frac{1}{n_{2}^{3}}\right) .
$$

We can now compare these results with the gravity prediction obtained in section 3.1. The massless supergravity vector fields $A^{i}, i=1,2,3$, are associated with the Cartan subalgebra $\mathrm{U}(1)^{3} \subset \mathrm{SO}(6)$. In particular, (3.12) implies

$$
\mathfrak{p}^{i}=2 g m^{i}=\frac{1}{3}\left(\frac{1}{n_{1}}-\frac{1}{n_{2}}\right), \quad \text { for } \quad i=1,2,3 .
$$


We also need the following relations among $5 \mathrm{~d}$ and $4 \mathrm{~d}$ quantities [3]

$$
\begin{aligned}
G_{\mathrm{N}}^{(5)} & =2 \pi G_{\mathrm{N}}^{(4)}, \quad J=\frac{1}{2 G_{\mathrm{N}}^{(4)}} \mathcal{J}, \quad Q_{0}=\frac{1}{2 \sqrt{2} G_{\mathrm{N}}^{(4)}} q_{0}, \\
Q_{i} & =\frac{1}{2 \sqrt{2} g_{(5)} G_{\mathrm{N}}^{(4)}} q_{i}, \quad \text { for } \quad i=1,2,3 .
\end{aligned}
$$

Finally, we will use the well-known holographic relation for $\mathrm{AdS}_{5} \times S^{5}$

$$
N^{2}=\frac{\pi}{2 g_{(5)}^{3} G_{\mathrm{N}}^{(5)}} .
$$

The constraint (3.19) can be now interpreted as the fact that the black spindle has charge zero with respect to the exact $R$-symmetry

$$
\stackrel{\circ}{R}=\sum_{i=1}^{3} \stackrel{\circ}{\Delta}_{i} Q_{i}+\stackrel{\circ}{\epsilon} J
$$

of the CFT. An analogous phenomenon was observed for the rotating black strings discussed in [3].

At this point, the entropy of the rotating black spindle (3.20) can be written as ${ }^{16}$

$$
\begin{aligned}
S_{\mathrm{BH}} & \equiv \log \rho_{\text {susy }}\left(Q_{0}, Q_{A}, J\right) \\
& =2 \pi \sqrt{\frac{c^{\mathrm{CFT}}}{6}\left(Q_{0}-\frac{1}{2} \sum_{A, B=1}^{2}\left(Q_{A}-Q_{3}\right)\left(k^{-1}\right)_{A B}\left(Q_{B}-Q_{3}\right)-\frac{J^{2}}{2 k}\right)},
\end{aligned}
$$

in complete agreement with the charged Cardy formula (4.1).

The charged Cardy formula can be reformulated as an extremization problem. The standard derivation of the Cardy formula extracts the density of states from the hightemperature behavior of the CFT partition function that is uniquely fixed by modular transformations [1]. Analogously, the asymptotic density of supersymmetric states can be extracted from the asymptotic behavior of the elliptic genus of the CFT, which is in turn fully determined by the 't Hooft anomalies of the theory. In our particular context, the density of states can be obtained via extremizing [3]

$$
\mathcal{I}_{\mathrm{CFT}}\left(\epsilon, \Delta_{i}\right)=\frac{\pi^{2}}{6 \beta} c_{r}\left(\Delta_{i}, \epsilon\right)+\beta Q_{0}+\mathrm{i} \pi \sum_{i=1}^{3} \Delta_{i} Q_{i}+\mathrm{i} \pi \epsilon J,
$$

with respect to $\beta, \epsilon, \Delta_{1,2,3}$, under the constraint (4.21). In this language, the constraint on charges (3.19) arises from imposing the reality condition

$$
\left.\llbracket \mathrm{m} \mathcal{I}_{\mathrm{CFT}}\right|_{\text {crit. }}=\mathrm{i} \pi\left(\sum_{i=1}^{3} \stackrel{\circ}{\Delta}_{i} Q_{i}+\stackrel{\circ}{\epsilon} J\right)=0,
$$

and we obtain $\log \rho_{\text {susy }}=\left.\operatorname{Re} \mathcal{I}_{\mathrm{CFT}}\right|_{\text {crit. }}$. We note that the functional (4.31) is the field theory counterpart of the entropy functional (3.47) based on gravitational blocks. Using (4.16) and (4.17), it is easy indeed to check that the two extremization problems can be mapped onto each other by identifying $\lambda^{\Lambda}$ with $\left(\beta, \Delta_{i}\right)$ (up to a suitable rescaling).

\footnotetext{
${ }^{16}$ Here, we absorbed the vacuum energy in the definition of $Q_{0}=n_{l}-\frac{c_{l}}{24}$.
} 


\subsection{The case with arbitrary magnetic charges}

In the case of arbitrary magnetic charges, the anomaly polynomial is given by

$$
\begin{aligned}
\mathcal{A}_{2 \mathrm{~d}}=a_{4 \mathrm{~d}} & \left(2\left(\Delta_{1} \Delta_{2} \mathfrak{p}^{3}+\Delta_{1} \Delta_{3} \mathfrak{p}^{2}+\Delta_{2} \Delta_{3} \mathfrak{p}^{1}\right) c_{1}\left(F_{R}\right)^{2}\right. \\
& +\left(\frac{1}{18}\left(\frac{1}{n_{1}}+\frac{1}{n_{2}}\right)\left(\frac{1}{n_{1}^{2}}-\frac{1}{n_{2}^{2}}\right)+\frac{1}{2} \mathfrak{p}^{1} \mathfrak{p}^{2} \mathfrak{p}^{3}\right) c_{1}(J)^{2} \\
& \left.-\frac{1}{3}\left(\frac{1}{n_{2}}+\frac{1}{n_{1}}\right)\left(\left(\Delta_{1}+\Delta_{2}\right) \mathfrak{p}^{3}+\left(\Delta_{1}+\Delta_{3}\right) \mathfrak{p}^{2}+\left(\Delta_{2}+\Delta_{3}\right) \mathfrak{p}^{1}\right) c_{1}\left(F_{R}\right) c_{1}(J)\right),
\end{aligned}
$$

with $\mathfrak{p}^{i}, i=1,2,3$, being the fluxes through the spindle for the $\mathrm{U}(1)^{3} \subset \mathrm{SO}(6)_{R}$ symmetry, satisfying

$$
\sum_{i=1}^{3} \mathfrak{p}^{i}=\frac{1}{n_{1}}-\frac{1}{n_{2}}
$$

By allowing a mixing $c_{1}(J)=\epsilon c_{1}\left(F_{R}\right)$ and extremizing the trial central charge $c_{r}\left(\epsilon, \Delta_{i}\right)=$ $6 \mathcal{A}_{2 \mathrm{~d}} / c_{1}\left(F_{R}\right)^{2}$ over the set of chemical potentials $\left(\epsilon, \Delta_{i}\right)$, under the constraint (4.21), we obtain the critical points

$$
\begin{aligned}
\stackrel{\circ}{\epsilon} & =\left(\frac{1}{n_{2}}-\frac{1}{n_{1}}\right)^{2} \frac{n_{1} n_{2}\left(n_{1}+n_{2}\right)}{n_{1} n_{2}\left(\left(\mathfrak{p}^{1}\right)^{2}+\left(\mathfrak{p}^{2}\right)^{2}+\left(\mathfrak{p}^{3}\right)^{2}\right)+\left(n_{1}^{2}+n_{2}^{2}\right)\left(\mathfrak{p}^{2} \mathfrak{p}^{3}+\mathfrak{p}^{1} \mathfrak{p}^{2}+\mathfrak{p}^{1} \mathfrak{p}^{3}\right)}, \\
\check{\triangle}_{1} & =\frac{1}{18}\left(\frac{1}{n_{2}}-\frac{1}{n_{1}}\right)^{2} \frac{3 n_{1} n_{2}\left(3 n_{2} \mathfrak{p}^{1}+2\right)-n_{1}^{2}\left(3 n_{2} \mathfrak{p}^{1}\left(6 n_{2} \mathfrak{p}^{1}+3\right)-3\right)+3 n_{2}^{2}}{n_{1} n_{2}\left(\left(\mathfrak{p}^{1}\right)^{2}+\left(\mathfrak{p}^{2}\right)^{2}+\left(\mathfrak{p}^{3}\right)^{2}\right)+\left(n_{1}^{2}+n_{2}^{2}\right)\left(\mathfrak{p}^{2} \mathfrak{p}^{3}+\mathfrak{p}^{1} \mathfrak{p}^{2}+\mathfrak{p}^{1} \mathfrak{p}^{3}\right)}, \\
\check{\Delta}_{2} & =\frac{1}{18}\left(\frac{1}{n_{2}}-\frac{1}{n_{1}}\right)^{2} \frac{3 n_{1} n_{2}\left(3 n_{2} \mathfrak{p}^{2}+2\right)-n_{1}^{2}\left(3 n_{2} \mathfrak{p}^{2}\left(6 n_{2} \mathfrak{p}^{2}+3\right)-3\right)+3 n_{2}^{2}}{n_{1} n_{2}\left(\left(\mathfrak{p}^{1}\right)^{2}+\left(\mathfrak{p}^{2}\right)^{2}+\left(\mathfrak{p}^{3}\right)^{2}\right)+\left(n_{1}^{2}+n_{2}^{2}\right)\left(\mathfrak{p}^{2} \mathfrak{p}^{3}+\mathfrak{p}^{1} \mathfrak{p}^{2}+\mathfrak{p}^{1} \mathfrak{p}^{3}\right)} .
\end{aligned}
$$

Using (4.5), we can then read off the exact central charge of the two-dimensional CFT

$$
c^{\mathrm{CFT}}=\frac{12 a_{4 \mathrm{~d}} \mathfrak{p}^{1} \mathfrak{p}^{2} \mathfrak{p}^{3}}{\frac{1}{n_{1} n_{2}}+\mathfrak{p}^{1} \mathfrak{p}^{2}+\mathfrak{p}^{3} \mathfrak{p}^{2}+\mathfrak{p}^{1} \mathfrak{p}^{3}} .
$$

Using (4.27), we can then write the entropy of the magnetically charged spindle (3.39) as

$$
S_{\mathrm{BH}} \equiv \log \rho_{\text {susy }}\left(Q_{0}\right)=2 \pi \sqrt{\frac{c^{\mathrm{CFT}}}{6} Q_{0}},
$$

in perfect agreement with the Cardy formula.

Notice, that for arbitrary magnetic charges, the flavor symmetries $K_{1}=Q_{1}-Q_{3}$, $K_{2}=Q_{2}-Q_{3}$ and $K_{3}=J$ mix in a non-trivial way. For completeness, we give the matrix level $k_{A B}, A, B=1,2,3$,

$$
\begin{aligned}
& k_{1,1}=4 a_{4 \mathrm{~d}} \mathfrak{p}^{2}, \quad k_{1,2}=2 a_{4 \mathrm{~d}}\left(\mathfrak{p}^{1}+\mathfrak{p}^{2}-\mathfrak{p}^{3}\right), \quad k_{1,3}=-\frac{1}{3} a_{4 \mathrm{~d}}\left(\frac{1}{n_{1}}+\frac{1}{n_{2}}\right)\left(\mathfrak{p}^{1}-\mathfrak{p}^{3}\right), \\
& k_{2,2}=4 a_{4 \mathrm{~d}} \mathfrak{p}^{1}, \quad k_{2,3}=-\frac{1}{3} a_{4 \mathrm{~d}}\left(\frac{1}{n_{1}}+\frac{1}{n_{2}}\right)\left(\mathfrak{p}^{2}-\mathfrak{p}^{3}\right), \\
& k_{3,3}=-a_{4 \mathrm{~d}}\left(\frac{1}{9}\left(\frac{1}{n_{1}}+\frac{1}{n_{2}}\right)\left(\frac{1}{n_{1}^{2}}-\frac{1}{n_{2}^{2}}\right)+\mathfrak{p}^{1} \mathfrak{p}^{2} \mathfrak{p}^{3}\right) .
\end{aligned}
$$




\section{$5 \mathcal{A}_{2 \mathrm{~d}}$ for general $\mathcal{N}=1$ theories on the spindle}

The black string solution in [12], being a solution to minimal gauged supergravity, can be embedded in all $\mathrm{AdS}_{5}$ string and M-theory compactifications, ${ }^{17}$ in particular in all the type IIB vacua $\mathrm{AdS}_{5} \times \mathrm{SE}_{5}$ associated with five-dimensional regular Sasaki-Einstein manifolds $\mathrm{SE}_{5}$. It would be interesting to find spindle black string solutions depending on more general charges and angular momentum in such compactifications. While finding the explicit solution is a difficult task, there is a simple quantum field theory prediction for the anomaly polynomial and the central charge of the corresponding $\mathrm{CFT}_{2}$. In this section, we briefly discuss the form of the anomaly polynomial for general theories. We also discuss the case of solutions of mimimal and half-maximal gauged supergravity that can be embedded in infinitely many type II and M theory compactifications.

\subsection{Integrating the anomaly polynomial}

Consider the compactification of a four-dimensional $\mathcal{N}=1$ SCFT with $d$ abelian global symmetries dual to $\mathrm{AdS}_{5} \times \mathrm{SE}_{5}$ on $\Sigma$. In the large $N$ limit, the $4 \mathrm{~d}$ anomaly polynomial can be written as

$$
\mathcal{A}_{4 \mathrm{~d}}=\frac{1}{6} \sum_{i, j, k=1}^{d} c_{i j k} c_{1}\left(F_{i}\right) c_{1}\left(F_{j}\right) c_{1}\left(F_{k}\right),
$$

where $F_{i}$ is a basis of $R$-symmetries with generators $Q_{i}$ and $c_{i j k}$ are related to the 't Hooft anomaly coefficients. In the toric case, there is a quite explicit description of the generators $Q_{i}$ on fields and the anomaly coefficients $c_{i j k}$ in terms of toric data [23, 41-44]. ${ }^{18}$ We turn on background gauge fields $A_{i}=\rho_{i}(p) \mathrm{d} \phi$ on the spindle with magnetic fluxes $\mathfrak{p}^{i}$ satisfying

$$
\sum_{i=1}^{d} \mathfrak{p}^{i}=\frac{1}{n_{1}}-\frac{1}{n_{2}}
$$

and work in the gauge

$$
\rho_{i}\left(p_{2}\right)=\frac{1}{2}\left(\mathfrak{p}^{i}-\frac{r_{0}^{i}}{2} \chi\right), \quad \rho_{i}\left(p_{1}\right)=\frac{1}{2}\left(-\mathfrak{p}^{i}-\frac{r_{0}^{i}}{2} \chi\right),
$$

where $\sum_{i=1}^{d} r_{0}^{i}=2$, which fixes the values of $R$-symmetry background field at the poles as in formula (4.10).

We also turn on background fields $A_{R}$ and $A_{J}$ for the $R$-symmetry and the internal $\mathrm{U}(1)$ isometry, with curvature

$$
F_{i}=\Delta_{i} F_{R}+\rho_{i}^{\prime}(p) \mathrm{d} p\left(\mathrm{~d} \phi+A_{J}\right)+\rho_{i}(p) F_{J}, \quad \text { for } \quad i=1,2, \ldots, d,
$$

where we have embedded the $2 \mathrm{~d} \mathrm{U}(1)_{R}$ symmetry in the direction $\Delta_{i} Q_{i}$ with $\sum_{i=1}^{d} \Delta_{i}=2$.

\footnotetext{
${ }^{17}$ There are restrictions due to the quantization conditions of the charges and the regularity of the uplift that need to be discussed on a case-by-case basis.

${ }^{18}$ We follow the conventions of [45], where more details can be found. In particular, $c_{i j k}=$ $\frac{N^{2}}{2}\left|\operatorname{det}\left(v_{i}, v_{j}, v_{k}\right)\right|$ where $v_{i}$ are the integer vectors defining the toric fan. For $\mathcal{N}=4 \mathrm{SYM}, c_{123}=N^{2} / 2$ and for the conifold $c_{123}=c_{124}=c_{234}=c_{134}=N^{2} / 2$. Notice that, in our normalizations, the $F_{i}$ assign charge +1 to the superpotential.
} 
The two-dimensional anomaly polynomial reads

$$
\mathcal{A}_{2 \mathrm{~d}}=\int_{\Sigma} \mathcal{A}_{4 \mathrm{~d}}
$$

Repeating the same arguments as in section 4.1, it is easy to see that the anomaly polynomial can be compactly written again as a gluing formula

$$
\mathcal{A}_{2 \mathrm{~d}}=\frac{16}{27 c_{1}(J)}\left(a_{4 \mathrm{~d}}\left(\Delta_{i}^{(1)}\right)-a_{4 \mathrm{~d}}\left(\Delta_{i}^{(2)}\right)\right),
$$

where

$$
\Delta_{i}^{(1)}=\Delta_{i} c_{1}\left(F_{R}\right)+\frac{c_{1}(J)}{2}\left(\mathfrak{p}^{i}-\frac{r_{0}^{i}}{2} \chi\right), \quad \Delta_{i}^{(2)}=\Delta_{i} c_{1}\left(F_{R}\right)-\frac{c_{1}(J)}{2}\left(\mathfrak{p}^{i}+\frac{r_{0}^{i}}{2} \chi\right)
$$

and we have defined the $4 \mathrm{~d}$ trial central charge in the large $N$ limit

$$
a_{4 \mathrm{~d}}\left(\Delta_{i}\right)=\frac{9}{32} \sum_{i, j, k=1}^{d} c_{i j k} \Delta_{i} \Delta_{j} \Delta_{k} .
$$

More explicitly, we can write

$$
\begin{aligned}
\mathcal{A}_{2 \mathrm{~d}}= & \frac{16}{27} \sum_{i=1}^{d} \frac{\partial a_{4 \mathrm{~d}}\left(\Delta_{i}\right)}{\partial \Delta_{i}} \mathfrak{p}^{i} c_{1}\left(F_{R}\right)^{2}-\frac{4 \chi}{27} \sum_{i, j=1}^{d} \frac{\partial^{2} a_{4 \mathrm{~d}}\left(\Delta_{i}\right)}{\partial \Delta_{i} \partial \Delta_{j}} \mathfrak{p}^{i} r_{0}^{j} c_{1}\left(F_{R}\right) c_{1}(J) \\
& +\frac{2}{81} \sum_{i, j, k=1}^{d} \frac{\partial^{3} a_{4 \mathrm{~d}}\left(\Delta_{i}\right)}{\partial \Delta_{i} \partial \Delta_{j} \partial \Delta_{k}}\left[\mathfrak{p}^{i} \mathfrak{p}^{j} \mathfrak{p}^{k}+\frac{3 \chi^{2}}{4} \mathfrak{p}^{i} r_{0}^{j} r_{0}^{k}\right] c_{1}(J)^{2} .
\end{aligned}
$$

By allowing a mixing $c_{1}(J)=\epsilon c_{1}\left(F_{R}\right)$ and extremizing the trial central charge $c_{r}\left(\epsilon, \Delta_{i}\right)=$ $6 \mathcal{A}_{2 \mathrm{~d}} / c_{1}\left(F_{R}\right)^{2}$ under the constraint $\sum_{i=1}^{d} \Delta_{i}=2$, we can determine the exact central charge of the $\mathrm{CFT}_{2}$.

Let us consider, for example, the Klebanov-Witten theory [46]. The manifold in this case is $Y_{5}=T^{1,1}$. The quiver contains two $\mathrm{SU}(N)$ gauge groups with two bi-fundamental chiral fields $A_{i}$ in the representation $(\mathbf{N}, \overline{\mathbf{N}})$ and two bi-fundamental chiral fields $B_{i}$ in the representation $(\overline{\mathbf{N}}, \mathbf{N})$ with a quartic superpotential

$$
W=\operatorname{Tr}\left(A_{1} B_{1} A_{2} B_{2}-A_{1} B_{2} A_{2} B_{1}\right) .
$$

We introduce four chemical potentials $\Delta_{I}$ and fluxes $\mathfrak{p}^{I}$, one for each of the four fields $\left\{A_{i}, B_{i}\right\}$, associated with the four global symmetries of the theory and satisfying

$$
\sum_{I=1}^{4} \Delta_{I}=2, \quad \sum_{I=1}^{4} \mathfrak{p}^{I}=\frac{1}{n_{1}}-\frac{1}{n_{2}} .
$$

The $4 \mathrm{~d}$ trial central charge in the large $N$ limit reads ${ }^{19}$

$$
a_{4 \mathrm{~d}}\left(\Delta_{i}\right)=\frac{27}{32} N^{2}\left(\Delta_{1} \Delta_{2} \Delta_{3}+\Delta_{1} \Delta_{4} \Delta_{3}+\Delta_{2} \Delta_{4} \Delta_{3}+\Delta_{1} \Delta_{2} \Delta_{4}\right) .
$$

\footnotetext{
${ }^{19}$ See footnote 18 for the 't Hooft anomaly coefficients.
} 
The exact $R$-symmetry corresponds to $\Delta_{I}=1 / 2$ and the exact central charge is given by

$$
a_{4 \mathrm{~d}}=\frac{27}{64} N^{2} .
$$

Using $r_{0}^{i}=1 / 2$, we can write the anomaly polynomial

$$
\begin{aligned}
\frac{27}{a_{4 \mathrm{~d}}} \mathcal{A}_{2 \mathrm{~d}}= & 32\left(\left(\Delta_{3} \Delta_{4}+\Delta_{2} \Delta_{3}+\Delta_{2} \Delta_{4}\right) \mathfrak{p}^{1}+\left(\Delta_{1} \Delta_{3}+\Delta_{1} \Delta_{4}+\Delta_{3} \Delta_{4}\right) \mathfrak{p}^{2}\right. \\
& \left.+\left(\Delta_{2} \Delta_{4}+\Delta_{1} \Delta_{2}+\Delta_{1} \Delta_{4}\right) \mathfrak{p}^{3}+\left(\Delta_{1} \Delta_{2}+\Delta_{1} \Delta_{3}+\Delta_{2} \Delta_{3}\right) \mathfrak{p}^{4}\right) c_{1}\left(F_{R}\right)^{2} \\
& +\frac{1}{2}\left(3\left(\frac{1}{n_{1}}-\frac{1}{n_{2}}\right)\left(\frac{1}{n_{1}}+\frac{1}{n_{2}}\right)^{2}+16 \sum_{I<J<K} \mathfrak{p}^{I} \mathfrak{p}^{J} \mathfrak{p}^{K}\right) c_{1}(J)^{2} \\
& -8\left(\frac{1}{n_{1}}+\frac{1}{n_{2}}\right) \sum_{I=1}^{4}\left(2-\Delta_{I}\right) \mathfrak{p}^{I} c_{1}\left(F_{R}\right) c_{1}(J),
\end{aligned}
$$

and, with the same method used in section 4.3, we can extract from it the exact central charge of the $\mathrm{CFT}_{2}$ and the levels of the various $\mathrm{U}(1)$ symmetries. Defining

$$
\Theta_{\mathrm{KW}}(\mathfrak{p})=\sum_{\substack{I<J \\(\neq K)}}^{4} \mathfrak{p}^{I} \mathfrak{p}^{J}\left(\mathfrak{p}^{K}\right)^{4}-2 \sum_{I, J=1}^{4} \mathfrak{p}^{I} \mathfrak{p}^{J} \prod_{K=1}^{4} \mathfrak{p}^{K}
$$

we find the exact central charge

$$
c^{\mathrm{CFT}}=\frac{64}{9} a_{4 \mathrm{~d}} \frac{\left(n_{1}-n_{2}\right)^{2}\left(\mathfrak{p}_{1} \mathfrak{p}_{2} \mathfrak{p}_{3}+\mathfrak{p}_{1} \mathfrak{p}_{4} \mathfrak{p}_{3}+\mathfrak{p}_{2} \mathfrak{p}_{4} \mathfrak{p}_{3}+\mathfrak{p}_{1} \mathfrak{p}_{2} \mathfrak{p}_{4}\right)}{\left(n_{1}^{2}+n_{1} n_{2}+n_{2}^{2}\right) \prod_{I<J}^{4}\left(\mathfrak{p}^{I}+\mathfrak{p}^{J}\right)+n_{1} n_{2} \Theta_{\mathrm{KW}}(\mathfrak{p})} .
$$

Furthermore, organizing the flavor symmetries in the basis $K_{i}=Q_{i}-Q_{4}$ for $i=1,2,3$ and $K_{4}=J$, we read the levels

$$
\begin{aligned}
& k_{1,1}=\frac{64}{27} a_{4 \mathrm{~d}}\left(\mathfrak{p}^{2}+\mathfrak{p}^{3}\right), \quad k_{2,2}=\frac{64}{27} a_{4 \mathrm{~d}}\left(\mathfrak{p}^{1}+\mathfrak{p}^{3}\right), \quad k_{3,3}=\frac{64}{27} a_{4 \mathrm{~d}}\left(\mathfrak{p}^{1}+\mathfrak{p}^{2}\right), \\
& k_{4,4}=-\frac{16}{27} a_{4 \mathrm{~d}}\left(\frac{3}{16}\left(\frac{1}{n_{1}}+\frac{1}{n_{2}}\right)\left(\frac{1}{n_{1}^{2}}-\frac{1}{n_{2}^{2}}\right)+\mathfrak{p}^{1} \mathfrak{p}^{2} \mathfrak{p}^{3}+\mathfrak{p}^{1} \mathfrak{p}^{4} \mathfrak{p}^{3}+\mathfrak{p}^{2} \mathfrak{p}^{4} \mathfrak{p}^{3}+\mathfrak{p}^{1} \mathfrak{p}^{2} \mathfrak{p}^{4}\right), \\
& k_{1,2}=k_{1,3}=k_{2,3}=\frac{32}{27} a_{4 \mathrm{~d}}\left(\mathfrak{p}^{1}+\mathfrak{p}^{2}+\mathfrak{p}^{3}-\mathfrak{p}^{4}\right), \\
& k_{4, j}=\frac{8}{27} a_{4 \mathrm{~d}}\left(\frac{1}{n_{1}}+\frac{1}{n_{2}}\right)\left(\mathfrak{p}^{4}-\mathfrak{p}^{j}\right), \quad \text { for } \quad j=1,2,3 .
\end{aligned}
$$

Notice that in our discussion we allowed a generic gauge for the flavor symmetries parameterized by the quantities $r_{0}^{i}$. One can choose, for example, the value $r_{0}^{i}=2 / d$ for all models. The effect of a change of gauge for the flavor symmetries can be reabsorbed by a shift of $\Delta_{i}$ in $c_{r}\left(\epsilon, \Delta_{i}\right)$ and leads to the same exact central charge $c^{\mathrm{CFT}}$. It leads however to a redefinition of charges. In particular, the charge $J$ associated with the internal isometry would be shifted by a linear combinations of the flavor charges. These redefinitions should be taken into account when computing the levels and writing the charged Cardy formula, but all physical conclusions are obviously unchanged. 


\subsection{The universal spindles in theories with 8 and 16 supercharges}

There are two simple universal cases where we can test (5.6) against supergravity predictions. As already mentioned, the stu model in five dimensions admits truncations to both minimal $\mathcal{N}=2$ and $\mathcal{N}=4$ gauged supergravites and the corresponding solutions can be uplifted to any $\mathrm{AdS}_{5}$ compactification with eight and sixteen supercharges, respectively (charge quantization conditions and regularity of the uplift allowing). This calls for a universal field theory counting of microstates in $\mathcal{N}=1$ and $\mathcal{N}=2$ SCFTs, in the spirit of $[7,47-49]$.

Let us consider first the case with eight supercharges, which is a straightforward generalization of the universal static case discussed in [12]. The rotating black spindle of section 3.1 when restricted to equal electric charges $q_{1}=q_{2}=q_{3}$ is a solution of minimal gauged supergravity and, as such, it can be embedded in all string and M-theory compactifications with an $\mathrm{AdS}_{5}$ vacuum and eight supercharges [29]. For all these solutions, the entropy takes the universal form

$$
S_{\mathrm{BH}} \equiv \log \rho_{\mathrm{susy}}\left(Q_{0}, J\right)=2 \pi \sqrt{\frac{c^{\mathrm{CFT}}}{6}\left(Q_{0}-\frac{J^{2}}{2 k}\right)},
$$

with

$$
c^{\mathrm{CFT}}=\frac{4 a_{4 \mathrm{~d}}}{3} \frac{\left(n_{2}-n_{1}\right)^{3}}{n_{1} n_{2}\left(n_{1}^{2}+n_{1} n_{2}+n_{2}^{2}\right)}, \quad k=-\frac{4 a_{4 \mathrm{~d}}}{27}\left(\frac{1}{n_{1}^{3}}-\frac{1}{n_{2}^{3}}\right),
$$

where $a_{4 \mathrm{~d}}=\frac{\pi}{8 G_{\mathrm{N}}^{(5)} g_{(5)}^{3}}$ is the exact central charge of the dual $\mathcal{N}=1$ SCFT. These solutions can be interpreted as universal compactifications on the spindle with angular momentum and magnetic charges aligned with the exact four-dimensional $R$-symmetry $\sum_{i=1}^{d} \bar{\Delta}_{i} Q_{i}$ of the $\mathcal{N}=1 \mathrm{SCFT}^{20}$

$$
\mathfrak{p}^{i}=\frac{1}{2}\left(\frac{1}{n_{1}}-\frac{1}{n_{2}}\right) \bar{\Delta}_{i}, \quad \text { for } \quad i=1,2, \ldots, d .
$$

It is easy to see that the anomaly polynomial of the two-dimensional CFT (5.6) collapses to the anomaly polynomial $(4.20)$ of $\mathcal{N}=4 \mathrm{SYM}$ with $\Delta_{i}=2 / 3,{ }^{21}$

$$
\mathcal{A}_{2 \mathrm{~d}}=\frac{2 a_{4 \mathrm{~d}}}{27}\left(12\left(\frac{1}{n_{1}}-\frac{1}{n_{2}}\right) c_{1}\left(F_{R}\right)^{2}+\left(\frac{1}{n_{1}^{3}}-\frac{1}{n_{2}^{3}}\right) c_{1}(J)^{2}-6\left(\frac{1}{n_{1}^{2}}-\frac{1}{n_{2}^{2}}\right) c_{1}\left(F_{R}\right) c_{1}(J)\right),
$$

as already computed in $[12,(30)]$. Notice that this expression only depends on $a_{4 \mathrm{~d}}$. The universal form of (5.18) then follows from the universality of the anomaly polynomial and the charged Cardy formula.

Consider now the case with sixteen supercharges, in the spirit of [49]. The fivedimensional uplift of the static spindle of section 3.2 when restricted to the magnetic

\footnotetext{
${ }^{20}$ Notice that the exact $R$-charges $\bar{\Delta}_{i}$ should satisfy some conditions for the construction to work, in particular they should be at least rational. This restrict the class of compactifications that can be used.

${ }^{21}$ The best way of doing this computation is to use the gauge $r_{0}^{i}=\bar{\Delta}_{i}$. Since there are no flavor charges and (5.6) is extremized at $\Delta_{i}=\bar{\Delta}_{i}$, we can restrict to $\Delta_{i}=\bar{\Delta}_{i}$. Recall that the exact four-dimensional $R$-symmetry $\bar{\Delta}_{i}$ is obtained by extremizing (5.8) and $a_{4 \mathrm{~d}}=\frac{9}{32} \sum_{i, j, k=1}^{d} c_{i j k} \bar{\Delta}_{i} \bar{\Delta}_{j} \bar{\Delta}_{k}$.
} 
charges $m^{2}=m^{3}$ are solutions of the half-maximal gauged supergravity in five dimensions. Such solutions can be embedded in all $\mathrm{AdS}_{5}$ type II or M-theory backgrounds with sixteen supercharges [26-28]. For these solutions the exact central charge of the corresponding $\mathrm{CFT}_{2}$ is given by

$$
c^{\mathrm{CFT}}=\frac{12 a_{4 \mathrm{~d}} \mathfrak{p}^{1}\left(\mathfrak{p}^{2}\right)^{2}}{\frac{1}{n_{1} n_{2}}+2 \mathfrak{p}^{1} \mathfrak{p}^{2}+\left(\mathfrak{p}^{2}\right)^{2}},
$$

where $\mathfrak{p}^{i}=2 g m^{i}$ and $\mathfrak{p}^{1}+2 \mathfrak{p}^{2}=\frac{1}{n_{1}}-\frac{1}{n_{2}}$. The universality of this formula follows again from the universality of the four-dimensional trial central charge for $\mathcal{N}=2$ SCFTs [50]. As discussed in [49], for all the theories with a holographic dual in the large $N$ limit we have

$$
a_{4 \mathrm{~d}}\left(\Delta_{i}\right)=\frac{27 a_{4 \mathrm{~d}}}{8} \Delta_{1} \Delta_{2}^{2}
$$

where $\Delta_{1}$ and $\Delta_{2}$, with $\Delta_{1}+2 \Delta_{2}=2$, are associated with the $\mathrm{U}(1)_{R}$ and the Cartan generator of the $\mathrm{SU}(2)_{R}$ symmetry, respectively. ${ }^{22}$ It is then straightforward to check that the anomaly polynomial of the two-dimensional CFT (5.6) collapses to the anomaly polynomial (4.20) of $\mathcal{N}=4 \mathrm{SYM}$ with $\Delta_{2}=\Delta_{3}$. The expression (5.22) then follows from the same computation as in section 4.3.

\section{Discussion and outlook}

In this paper we constructed supersymmetric near-horizon solutions describing dyonic rotating spindle black strings that can be embedded in $\mathrm{AdS}_{5} \times S^{5}$ and successfully matched the corresponding density of states with the charged Cardy formula. Many questions are left open.

First of all, we were able to construct horizon geometries only. Given the successful field theory analysis based on $\mathcal{N}=4 \mathrm{SYM}$, we expect these solutions to arise as supersymmetric domain walls that interpolate between the near horizon region and $\mathrm{AdS}_{5}$. It would be interesting to construct the full interpolating solution by generalizing the ansatz in [14]. Similarly, it would be interesting to find spindle solutions in more general compactifications than $\mathrm{AdS}_{5} \times S^{5}$. The case of universal solutions has been discussed in section 5.2. More general solutions are more difficult to find. In order to use gauged supergravity, we need the existence of a consistent truncation and deal with supergravity theories with hypermultiplets. Some recent examples in the context of AdS black strings obtained from twisted compactifications can be found in [3].

Another important question concerns the field theory interpretation of the spindle compactifications. From the four-dimensional point of view, we are considering a SCFT on a singular manifold. It would be interesting to understand whether this makes sense as a field theory in the presence of defects or it has an interpretation as the reduction of a more complicated quantum field theory on a higher-dimensional smooth manifold.

\footnotetext{
${ }^{22}$ In a perturbative theory the $4 \mathrm{~d}$ trial $R$-symmetry is $R(\Delta)=\Delta_{1} r_{1} / 2+\Delta_{2} r_{2}$, where $r_{1}$ assigns charge 2 to the chiral field $\phi_{I}$ in the vector multiplet and zero to the chiral pairs $q_{a}, \tilde{q}_{a}$ in a hypermultiplet and $r_{2}$ assigns charge zero to $\phi_{I}$ and charge 1 to $q_{a}, \tilde{q}_{a}$. Compared with [49] we rescaled $\Delta_{2}^{\text {there }}=2 \Delta_{2}^{\text {here }}$, for ease of comparison with $\mathcal{N}=4 \mathrm{SYM}$.
} 
We have seen that the anomaly polynomial of the low-energy CFT in two dimensions computed from purely four-dimensional data correctly reproduces the holographic results in the bulk. On the other hand, the anomaly polynomial of the two-dimensional CFT should be also computable from a ten-dimensional point of view using the anomaly inflow method developed in [51]. This could not only put the computation on firmer ground but it could also shed some light on the physics of the system.

Another natural question is whether the anomaly and other physical quantities, in particular the elliptic genus of the $\mathrm{CFT}_{2}$, can be encoded in a higher-dimensional supersymmetric index, which could give information also at finite $N$. In the analogous case of compactifications on the sphere, the relevant quantity is the refined topologically twisted index [52], which is the partition function on $T^{2} \times S^{2}$ with a topological twist and an $\Omega$-background along $S^{2}$. This has been explicitly discussed in $[2,53]$. Here the relevant index should be associated with a refined partition function on $T^{2} \times \mathbb{W} \mathbb{P}_{\left[n_{1}, n_{2}\right]}^{1}$, with an appropriate prescription for dealing with the singularities. We notice that the supersymmetry on the spindle is not realized with a topological twist, but it is still supported by a magnetic flux (3.14). The gluing formulae (3.47) and (5.6) suggest that the relevant index for the spindle could be obtained by fusing two copies of the four-dimensional holomorphic block [18] with fluxes $1 / 2 n_{1}$ and $1 / 2 n_{2}$ for the $R$-symmetry, respectively.

Finally, we notice that there exist also supersymmetric accelerating and spinning black holes with conical singularities in four dimensions. These can be interpreted as domain walls that interpolate between (conformal) $\mathrm{AdS}_{4}$ and a warped product of $\mathrm{AdS}_{2}$ and the spindle and can be embedded in $\mathrm{AdS}_{4} \times S^{7}$ [19]. There are many analogies with the situation considered in this paper. The near-horizon geometries can be found again with the formalism in [14]. Moreover, it is easy to check that an entropy functional for these black holes can be written by gluing two gravitational blocks with a formula similar to (3.47) using the prepotential $\mathcal{F}_{4 \mathrm{~d}}=\sqrt{X^{0} X^{1} X^{2} X^{3}}$. This corresponds to the familiar fact that, while the entropy of supersymmetric black objects in $\mathrm{AdS}_{5}$ is controlled by anomalies and the central charge $a_{4 \mathrm{~d}}(\Delta)$ of the dual four-dimensional SCFT, the entropy of black holes in $\mathrm{AdS}_{4}$ is controlled by the free energy on $S^{3}$ of the dual three-dimensional SCFT, which for the ABJM theory [54] reads $F_{S^{3}}=\frac{4 \pi \sqrt{2} k^{1 / 2} N^{3 / 2}}{3} \sqrt{\Delta_{0} \Delta_{1} \Delta_{2} \Delta_{3}}$ in the large $N$ limit [55]. This again suggests the possible existence of an index obtained by fusing two copies of the three-dimensional holomorphic block with fluxes $1 / 2 n_{1}$ and $1 / 2 n_{2}$ for the $R$-symmetry.

The present work, taken together with $[12,19]$, also raises a more general question outside the realm of holography and microscopic entropy counting. We already noted that the solutions with conical singularities actually represent the most generic cases in lowerdimensional supergravity theories with a non-vanishing scalar potential, where no special restrictions are imposed on the black hole geometry and asymptotic charges. The fact that we can make sense of the supersymmetric solutions holographically might suggest the interesting possibility that the spindle horizons are also relevant for more realistic thermal black hole models.

We leave all these questions for future work. 


\section{Acknowledgments}

SMH is supported in part by WPI Initiative, MEXT, Japan at IPMU, the University of Tokyo, JSPS KAKENHI Grant-in-Aid (Wakate-A), No.17H04837 and JSPS KAKENHI Grant-in-Aid (Early-Career Scientists), No.20K14462. KH is supported in part by the Bulgarian NSF grants DN08/3, N28/5, and KP-06-N 38/11. AZ is partially supported by the INFN, the ERC-STG grant 637844-HBQFTNCER, and the MIUR-PRIN contract $2017 \mathrm{CC} 72 \mathrm{MK} 003$.

Open Access. This article is distributed under the terms of the Creative Commons Attribution License (CC-BY 4.0), which permits any use, distribution and reproduction in any medium, provided the original author(s) and source are credited.

\section{References}

[1] J.L. Cardy, Operator Content of Two-Dimensional Conformally Invariant Theories, Nucl. Phys. B 270 (1986) 186 [INSPIRE].

[2] S.M. Hosseini, K. Hristov and A. Zaffaroni, Microstates of rotating AdS $S_{5}$ strings, JHEP 11 (2019) 090 [arXiv: 1909.08000] [inSPIRE].

[3] S.M. Hosseini, K. Hristov, Y. Tachikawa and A. Zaffaroni, Anomalies, Black strings and the charged Cardy formula, JHEP 09 (2020) 167 [arXiv: 2006.08629] [INSPIRE].

[4] J.M. Maldacena and C. Núñez, Supergravity description of field theories on curved manifolds and a no go theorem, Int. J. Mod. Phys. A 16 (2001) 822 [hep-th/0007018] [InSPIRE].

[5] F. Benini and N. Bobev, Exact two-dimensional superconformal R-symmetry and c-extremization, Phys. Rev. Lett. 110 (2013) 061601 [arXiv:1211.4030] [INSPIRE].

[6] F. Benini and N. Bobev, Two-dimensional SCFTs from wrapped branes and c-extremization, JHEP 06 (2013) 005 [arXiv: 1302.4451] [INSPIRE].

[7] F. Benini, N. Bobev and P.M. Crichigno, Two-dimensional SCFTs from D3-branes, JHEP 07 (2016) 020 [arXiv: 1511.09462] [INSPIRE].

[8] J.P. Gauntlett, O.A.P. Mac Conamhna, T. Mateos and D. Waldram, Supersymmetric AdS solutions of type IIB supergravity, Phys. Rev. Lett. 97 (2006) 171601 [hep-th/0606221] [INSPIRE].

[9] J.P. Gauntlett, N. Kim and D. Waldram, Supersymmetric $A d S_{3}, A d S_{2}$ and Bubble Solutions, JHEP 04 (2007) 005 [hep-th/0612253] [INSPIRE].

[10] H.K. Kunduri, J. Lucietti and H.S. Reall, Do supersymmetric anti-de Sitter black rings exist?, JHEP 02 (2007) 026 [hep-th/0611351] [INSPIRE].

[11] H.K. Kunduri and J. Lucietti, Near-horizon geometries of supersymmetric AdS $S_{5}$ black holes, JHEP 12 (2007) 015 [arXiv:0708.3695] [INSPIRE].

[12] P. Ferrero, J.P. Gauntlett, J.M. Pérez Ipiña, D. Martelli and J. Sparks, D3-branes Wrapped on a Spindle, Phys. Rev. Lett. 126 (2021) 111601 [arXiv:2011.10579] [InSPIRE].

[13] K. Hristov, S. Katmadas and C. Toldo, Rotating attractors and BPS black holes in AdS , JHEP 01 (2019) 199 [arXiv:1811.00292] [INSPIRE]. 
[14] K. Hristov, S. Katmadas and C. Toldo, Matter-coupled supersymmetric Kerr-Newman-AdS 4 black holes, Phys. Rev. D 100 (2019) 066016 [arXiv:1907.05192] [InSPIRE].

[15] I. Bah, F. Bonetti, R. Minasian and E. Nardoni, Anomalies of QFTs from M-theory and Holography, JHEP 01 (2020) 125 [arXiv:1910.04166] [INSPIRE].

[16] I. Bah and F. Bonetti, Anomaly Inflow, Accidental Symmetry, and Spontaneous Symmetry Breaking, JHEP 01 (2020) 117 [arXiv:1910.07549] [INSPIRE].

[17] S.M. Hosseini, K. Hristov and A. Zaffaroni, Gluing gravitational blocks for AdS black holes, JHEP 12 (2019) 168 [arXiv:1909.10550] [INSPIRE].

[18] C. Beem, T. Dimofte and S. Pasquetti, Holomorphic Blocks in Three Dimensions, JHEP 12 (2014) 177 [arXiv:1211.1986] [INSPIRE].

[19] P. Ferrero, J.P. Gauntlett, J.M.P. Ipiña, D. Martelli and J. Sparks, Accelerating Black Holes and Spinning Spindles, arXiv:2012.08530 [INSPIRE].

[20] A. Gnecchi, K. Hristov, D. Klemm, C. Toldo and O. Vaughan, Rotating black holes in $4 d$ gauged supergravity, JHEP 01 (2014) 127 [arXiv:1311.1795] [INSPIRE].

[21] D. Klemm, Four-dimensional black holes with unusual horizons, Phys. Rev. D 89 (2014) 084007 [arXiv: 1401 .3107] [INSPIRE].

[22] S. Ferrara and A. Zaffaroni, $N=1, N=2$ 4-D superconformal field theories and supergravity in $A d S_{5}$, Phys. Lett. B 431 (1998) 49 [hep-th/9803060] [InSPIRE].

[23] S. Benvenuti, L.A. Pando Zayas and Y. Tachikawa, Triangle anomalies from Einstein manifolds, Adv. Theor. Math. Phys. 10 (2006) 395 [hep-th/0601054] [INSPIRE].

[24] H. Looyestijn, E. Plauschinn and S. Vandoren, New potentials from Scherk-Schwarz reductions, JHEP 12 (2010) 016 [arXiv:1008.4286] [INSPIRE].

[25] M. Cvetič et al., Embedding AdS black holes in ten-dimensions and eleven-dimensions, Nucl. Phys. B 558 (1999) 96 [hep-th/9903214] [INSPIRE].

[26] J.P. Gauntlett and O. Varela, $D=5 \mathrm{SU}(2) \times \mathrm{U}(1)$ Gauged Supergravity from $D=11$ Supergravity, JHEP 02 (2008) 083 [arXiv:0712.3560] [INSPIRE].

[27] D. Cassani, G. Josse, M. Petrini and D. Waldram, Systematics of consistent truncations from generalised geometry, JHEP 11 (2019) 017 [arXiv:1907.06730] [INSPIRE].

[28] E. Malek, Half-Maximal Supersymmetry from Exceptional Field Theory, Fortsch. Phys. 65 (2017) 1700061 [arXiv:1707.00714] [INSPIRE].

[29] J.P. Gauntlett and O. Varela, Consistent Kaluza-Klein reductions for general supersymmetric AdS solutions, Phys. Rev. D 76 (2007) 126007 [arXiv:0707.2315] [INSPIRE].

[30] L. Andrianopoli, S. Ferrara and M.A. Lledó, Scherk-Schwarz reduction of D $=5$ special and quaternionic geometry, Class. Quant. Grav. 21 (2004) 4677 [hep-th/0405164] [INSPIRE].

[31] D. Gaiotto, A. Strominger and X. Yin, New connections between 4-D and 5-D black holes, JHEP 02 (2006) 024 [hep-th/0503217] [INSPIRE].

[32] K. Behrndt, G. Lopes Cardoso and S. Mahapatra, Exploring the relation between 4-D and 5-D BPS solutions, Nucl. Phys. B 732 (2006) 200 [hep-th/0506251] [INSPIRE].

[33] G. Lopes Cardoso, J.M. Oberreuter and J. Perz, Entropy function for rotating extremal black holes in very special geometry, JHEP 05 (2007) 025 [hep-th/0701176] [INSPIRE]. 
[34] P. Meessen and T. Ortín, Supersymmetric solutions to gauged $N=2 d=4$ SUGRA: the full timelike shebang, Nucl. Phys. B 863 (2012) 65 [arXiv: 1204.0493] [InSPIRE].

[35] L. Andrianopoli et al., $N=2$ supergravity and $N=2$ superYang-Mills theory on general scalar manifolds: Symplectic covariance, gaugings and the momentum map, J. Geom. Phys. 23 (1997) 111 [hep-th/9605032] [INSPIRE].

[36] S.M. Hosseini, K. Hristov and A. Zaffaroni, An extremization principle for the entropy of

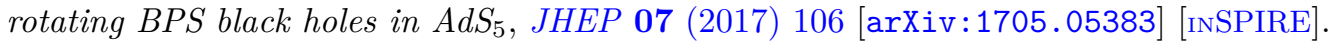

[37] F. Benini, E. Colombo, S. Soltani, A. Zaffaroni and Z. Zhang, Superconformal indices at large $N$ and the entropy of $A d S_{5} \times S E_{5}$ black holes, Class. Quant. Grav. 37 (2020) 215021 [arXiv: 2005.12308] [INSPIRE].

[38] S.L. Cacciatori, D. Klemm, D.S. Mansi and E. Zorzan, All timelike supersymmetric solutions of $N=2, D=4$ gauged supergravity coupled to abelian vector multiplets, JHEP 05 (2008) 097 [arXiv: 0804.0009] [INSPIRE].

[39] S.L. Cacciatori and D. Klemm, Supersymmetric AdS 4 black holes and attractors, JHEP 01 (2010) 085 [arXiv:0911.4926] [INSPIRE].

[40] N. Halmagyi, Static BPS black holes in AdS 4 with general dyonic charges, JHEP 03 (2015) 032 [arXiv: 1408.2831] [INSPIRE].

[41] S. Franco, A. Hanany, K.D. Kennaway, D. Vegh and B. Wecht, Brane dimers and quiver gauge theories, JHEP 01 (2006) 096 [hep-th/0504110] [INSPIRE].

[42] B. Feng, Y.-H. He, K.D. Kennaway and C. Vafa, Dimer models from mirror symmetry and quivering amoebae, Adv. Theor. Math. Phys. 12 (2008) 489 [hep-th/0511287] [INSPIRE].

[43] A. Butti and A. Zaffaroni, R-charges from toric diagrams and the equivalence of a-maximization and Z-minimization, JHEP 11 (2005) 019 [hep-th/0506232] [INSPIRE].

[44] S. Benvenuti and M. Kruczenski, From Sasaki-Einstein spaces to quivers via BPS geodesics: $L^{p, q \mid r}, J H E P 04$ (2006) 033 [hep-th/0505206] [INSPIRE].

[45] S.M. Hosseini and A. Zaffaroni, Proving the equivalence of c-extremization and its gravitational dual for all toric quivers, JHEP 03 (2019) 108 [arXiv:1901.05977] [InSPIRE].

[46] I.R. Klebanov and E. Witten, Superconformal field theory on three-branes at a Calabi-Yau singularity, Nucl. Phys. B 536 (1998) 199 [hep-th/9807080] [INSPIRE].

[47] F. Azzurli, N. Bobev, P.M. Crichigno, V.S. Min and A. Zaffaroni, A universal counting of

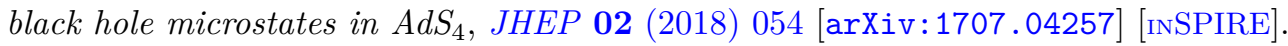

[48] N. Bobev and P.M. Crichigno, Universal RG Flows Across Dimensions and Holography, JHEP 12 (2017) 065 [arXiv:1708.05052] [INSPIRE].

[49] S.M. Hosseini and A. Zaffaroni, Universal AdS Black Holes in Theories with 16 Supercharges and Their Microstates, Phys. Rev. Lett. 126 (2021) 171604 [arXiv: 2011.01249] [INSPIRE].

[50] A.D. Shapere and Y. Tachikawa, Central charges of $N=2$ superconformal field theories in four dimensions, JHEP 09 (2008) 109 [arXiv:0804.1957] [INSPIRE].

[51] I. Bah, F. Bonetti, R. Minasian and P. Weck, Anomaly Inflow Methods for SCFT Constructions in Type IIB, JHEP 02 (2021) 116 [arXiv: 2002.10466] [INSPIRE].

[52] F. Benini and A. Zaffaroni, A topologically twisted index for three-dimensional supersymmetric theories, JHEP 07 (2015) 127 [arXiv: 1504.03698] [INSPIRE]. 
[53] S.M. Hosseini, A. Nedelin and A. Zaffaroni, The Cardy limit of the topologically twisted index and black strings in AdS $S_{5}$, JHEP 04 (2017) 014 [arXiv:1611.09374] [INSPIRE].

[54] O. Aharony, O. Bergman, D.L. Jafferis and J. Maldacena, $N=6$ superconformal Chern-Simons-matter theories, M2-branes and their gravity duals, JHEP 10 (2008) 091 [arXiv:0806.1218] [INSPIRE].

[55] D.L. Jafferis, I.R. Klebanov, S.S. Pufu and B.R. Safdi, Towards the F-Theorem: $N=2$ Field Theories on the Three-Sphere, JHEP 06 (2011) 102 [arXiv:1103.1181] [INSPIRE]. 